\title{
Macrophage deficiency of p38 $\alpha$ MAPK promotes apoptosis and plaque necrosis in advanced atherosclerotic lesions in mice
}

\author{
Tracie A. Seimon, ${ }^{1}$ Yibin Wang, ${ }^{2}$ Seongah Han, ${ }^{1}$ Takafumi Senokuchi, ${ }^{1}$ \\ Dorien M. Schrijvers, ${ }^{1}$ George Kuriakose, ${ }^{1}$ Alan R. Tall, ${ }^{1}$ and Ira A. Tabas ${ }^{1,3}$ \\ 1Department of Medicine, Columbia University, New York, New York, USA. \\ 2Department of Anesthesiology and Department of Medicine, UCLA, Los Angeles, California, USA \\ ${ }^{3}$ Department of Pathology and Cell Biology and Department of Physiology and Cellular Biophysics, Columbia University, New York, New York, USA.
}

\begin{abstract}
ER stress occurs in macrophage-rich areas of advanced atherosclerotic lesions and contributes to macrophage apoptosis and subsequent plaque necrosis. Therefore, signaling pathways that alter ER stress-induced apoptosis may affect advanced atherosclerosis. Here we placed Apoe ${ }^{-/-}$mice deficient in macrophage $\mathrm{p} 38 \alpha$ MAPK on a Western diet and found that they had a marked increase in macrophage apoptosis and plaque necrosis. The macrophage $\mathrm{p} 38 \alpha$-deficient lesions also exhibited a significant reduction in collagen content and a marked thinning of the fibrous cap, which suggests that plaque progression was advanced in these mice. Consistent with our in vivo data, we found that ER stress-induced apoptosis in cultured primary mouse macrophages was markedly accelerated under conditions of $\mathrm{p} 38$ inhibition. Pharmacological inhibition or genetic ablation of $\mathrm{p} 38$ suppressed activation of Akt in cultured macrophages and in atherosclerotic lesions. In addition, inhibition of Akt enhanced ER stress-induced macrophage apoptosis, and expression of a constitutively active myristoylated Akt blocked the enhancement of ER stress-induced apoptosis that occurred with p38 inhibition in cultured cells. Our results demonstrate that p38 $\alpha$ MAPK may play a critical role in suppressing ER stress-induced macrophage apoptosis in vitro and advanced lesional macrophage apoptosis in vivo.
\end{abstract}

\section{Introduction}

Recent studies have implicated p38 MAPK and its downstream target MK2 as potentially important mediators in the inflammatory response and atherosclerosis (1-4). Activation of p38 requires phosphorylation on both $\mathrm{Tyr}^{182}$ and $\mathrm{Thr}^{180}$ by the 2 upstream kinases, MKK3 and MKK6 (5). The role of p38 in regulating inflammation was initially discovered in a screen for proteins that modulate cytokine production during endotoxin stimulation (6). Since this discovery, p38 has been shown to play an important role in stimulating inflammatory cytokine production, endotoxin-induced shock, and arthritis (7-9). Because MK2 and p38 have been shown to play a central role in the production of proinflammatory mediators, these molecules have become attractive drug targets for the treatment of diseases such as rheumatoid arthritis and atherosclerosis (1).

Atherosclerotic plaques that are prone to disruption and acute luminal thrombosis are characterized by the combination of large necrotic areas, fibrous cap thinning, centralized apoptosis around the necrotic core, and high levels of inflammatory cytokines and matrix proteases (10-13). These dangerous plaques, including those in human coronary arteries, are also characterized by cells undergoing ER stress signaling (14-17).

ER stress signaling represents a response of cells to a wide variety of ER perturbations. These ER perturbations result in the accumulation of misfolded proteins and the induction of the unfolded

Conflict of interest: The authors have declared that no conflict of interest exists.

Nonstandard abbreviations used: acetyl-LDL, acetylated LDL; CHOP, C/EBP homologous protein; LysMCre, lysozyme-driven Cre recombinase; Myr-Akt, myristoylated Akt; PRR, pattern recognition receptor.

Citation for this article: J. Clin. Invest. 119:886-898 (2009). doi:10.1172/JCI37262. protein response (UPR). ER stress by design is an adaptive survival pathway that protects cells from the buildup of misfolded proteins. The ER stress pathway maintains protein quality control by halting global protein translation while simultaneously inducing UPR-regulated transcription factors and chaperones such as $\mathrm{C} / \mathrm{EBP}$ homologous protein (CHOP), ATF3, and GRP78 to correct the defect in protein folding. Several studies have demonstrated that ER stress is a prominent feature of lesional macrophages in atheromata from humans and mice, and ER-stressed macrophages reside in areas that have a high abundance of TUNEL-positive cells $(14,16,17)$. Prior work in our laboratory has shown that ER stress signaling can also enable advanced lesional macrophage apoptosis $(15,18-20)$. Macrophage death in this setting can lead directly to plaque necrosis $(12,21)$. Thus, factors that influence ER stress-induced apoptosis are likely to have important effects on advanced plaque progression.

Recent work has shown that p38 MAPK is activated under conditions of ER stress and in vivo during atherogenesis $(2,22,23)$. Although p38 MAPK has been shown to play a crucial role in regulating apoptosis, p38 can have both pro- and antiapoptotic functions depending on the apoptotic stimulus (2, 23-25). We recently tested the effect of inhibition or deficiency of p38 on a particular model of ER stress-induced macrophage death that may be relevant to advanced plaques (2). In this model, the macrophages were overloaded with lipoprotein-derived unesterified cholesterol, which triggers ER stress $(18,26)$. ER stress, together with activation of pattern recognition receptors (PRRs) by the lipoproteins themselves, triggers apoptosis $(2,27)$. In the present study, using this model, we observed that p38 inhibition or deficiency protected the cells from apoptosis, suggesting that p38 deficiency in vivo would suppress advanced plaque progression. 


\section{Table 1}

Metabolic characteristics of $p 38 a^{f / t / t} A p o e^{-/}$and $p 38 a^{f / f / t}$ LysM Cret/-Apoe $-/-$ mice fed a Western-type diet for 9 wk

\begin{tabular}{|c|c|c|c|}
\hline & $p 38 a^{f / f I} A p o e^{-/-}$ & p38a $a^{t / f / I} L_{s} M C r e^{+/-A p o e^{-/-}}$ & $P$ \\
\hline Total cholesterol (mg/dl) & $455 \pm 42(9)$ & $467 \pm 75(10)$ & 0.88 \\
\hline Triglyceride (mg/dl) & $73 \pm 11(8)$ & $72 \pm 5(7)$ & 0.89 \\
\hline Body weight before diet $(\mathrm{g})$ & $19.8 \pm 0.6(9)$ & $19.5 \pm 0.5(10)$ & 0.72 \\
\hline Body weight after diet (g) & $21.1 \pm 1.0(9)$ & $20.2 \pm 0.6(10)$ & 0.42 \\
\hline
\end{tabular}

Values are mean $\pm \mathrm{SEM} ; n$ is shown in parentheses.

To test this hypothesis, we used a cre-lox system to test the effect of macrophage p $38 \alpha$ deficiency on plaque morphology in Western diet-fed $A p o e^{-/-}$mice. To our surprise, we found that macrophage apoptosis and plaque progression were actually enhanced in these mice compared with Apoe $e^{-/}$mice. A more detailed examination of potential mechanisms in cultured macrophages revealed that in other atherosclerosis-relevant models of ER-stressed macrophages, p38 $\alpha$ deficiency promoted apoptosis by suppressing the Akt cell survival pathway. These data demonstrate an important prosurvival role for $\mathrm{p} 38$ in protecting from macrophage apoptosis and advanced plaque necrosis during atherosclerosis.

\section{Results}

Macrophage $p 38 \alpha$ deficiency leads to enhanced macrophage apoptosis and other markers of advanced plaque progression. Genetic deletion of $\mathrm{p} 38 \alpha$ MAPK in mice leads to an embryonic-lethal phenotype (28). To test the effect of macrophage p $38 \alpha$ on the development of atherosclerosis, we generated $p 38 a^{f l / f l}$ and $p 38 a^{f l / f l} \mathrm{LysMCr}^{+/-}$mice, the latter of which express lysozyme-driven Cre recombinase (LysMCre), as described previously (2). These mice were then bred into the Apoe $e^{-/-}$background to generate control $p 38 a^{f l / f l} \mathrm{Apoe}^{-/-}$(wild-type $\mathrm{p} 38 \alpha$ ) and $p 38 a^{\text {fl/fl}} \mathrm{LysMCr}^{+/-}$Apoe $\mathrm{e}^{-/-}$(macrophage p38 $\alpha$-deficient) mice in the C57BL/ 6 background, which were fed a Western-type diet for $9 \mathrm{wk}$. At the time of sacrifice, mice were analyzed for lipoprotein levels and macrophage p $38 \alpha$ expression. There was no statistically significant difference in body weight between groups before or after the diet (Table 1). The total cholesterol and triglyceride levels were also similar (Table 1). FPLC analysis showed that the VLDL fraction increased slightly and the IDL/LDL fraction decreased slightly in the $p 38 a^{f l / f l} \mathrm{LysMCre}^{+/-} \mathrm{Apoe}^{-/-}$mice (Figure 1A). To determine whether expression of LysMCre led to the loss of $\mathrm{p} 38 \alpha$ protein, peritoneal macrophages were harvested and analyzed for protein expression. Expression of p38 was completely absent in the $p 38 a^{f l / f l} \mathrm{LysMCr}^{+/-}$Apoe-/- macrophages (Figure 1B), consistent with our previous results (2). To confirm that macrophages in atherosclerotic lesions of $p 38 a^{f l f l} \mathrm{LysMCr}^{+/-} \mathrm{Apoe}^{-/-}$mice were also deficient in p38, we performed immunohistochemistry (Figure 1C). Aortic roots from $p 38 a^{f l / f l} \mathrm{Apoe}^{-/-}$mice exhibited robust staining of $\mathrm{p} 38$ in lesional areas that were also positive for the macrophage-specific marker AIA31240. In contrast, p38 staining, but not AIA31240 staining, was markedly reduced in aortic sections from $p 38 a^{f l f l}$ LysMCre $e^{+-} A p o e^{-/-}$mice. Macrophage staining was similar between both groups of mice (Figure 1D). Thus, LysMCre was functional in the atherosclerotic plaque.

We next quantified total lesion area in the aortic roots of both groups of mice and found no significant difference between the p38a fl/fl Apoe $e^{-/-}$and $p 38 a^{f / f l}$ LysMCre $e^{+/-}$Apoe $^{-/-}$lesions (Figure 2A). Remarkably, however, we found a dramatic increase in the mean necrotic area of the $p 38 a^{f l / f l} \mathrm{LysMCr}^{+/-} \mathrm{Apoe}^{-/-}$lesions $\left(46,700 \mu \mathrm{m}^{2}\right.$ compared with $18,965 \mu \mathrm{m}^{2}$ in control mice; $P<0.01$; Figure $2 \mathrm{~B})$ and a 2.2-fold increase in the percent necrotic area of the p38afl/fl LysMCre ${ }^{+/-}$Apoe $^{-/-}$lesions compared with control lesions (Figure 2C). Representative images of the enlarged necrotic areas present in the $p 38 a^{f / f l} \mathrm{LysMCre} \mathrm{Cr}^{+/-} \mathrm{Apoe} \mathrm{e}^{-/-}$lesions are shown in Figure $2 \mathrm{D}$, and areas defined and quantified as necrotic are shown in Figure $2 \mathrm{E}$. Thus, opposite to what we originally predicted, macrophage p38 $\alpha$ deficiency promotes plaque necrosis in this mouse model, indicating that $\mathrm{p} 38 \mathrm{MAPK}$ plays a protective role in this process.

Plaque necrosis is a consequence of advanced lesion macrophage apoptosis $(12,21)$. We therefore assessed the effect of p38 $\alpha$ deficiency on lesional macrophage apoptosis using TUNEL analysis and found that TUNEL-positive cells colocalized with nuclei in $p 38 a^{f l / f l} \mathrm{Apoe}^{-/-}$and $p 38 a^{f l / f l} \mathrm{LysMCre}{ }^{+/-} \mathrm{Apoe}^{-/-}$lesions (Figure 3A, arrows). We also observed TUNEL-positive staining within the developing necrotic core. Quantification of the data revealed an approximate $51 \%$ increase in the percent of apoptotic cells in the p38al/fl LysMCre ${ }^{+/-} \mathrm{Apoe}^{-/-}$lesions (Figure 3B). Similar results were observed when the lesions were stained for activated or cleaved caspase 3 (Figure 3C).

An alternative explanation that may account for the increase in apoptotic macrophages could be a defect in phagocytosis of apoptotic cells, a process known as efferocytosis. In this scenario, the steady-state level of apoptosis would be similar between both groups of mice. However, a defect in efferocytosis could lead to the observed accumulation of apoptotic cells in plaque. To determine whether macrophage p38 deficiency causes a reduction in efferocytosis, an in vivo efferocytosis assay was performed (see Methods). As shown in Figure 3D, p38-deficient macrophages ingested apoptotic cells as efficiently as did controls. Taken together, these data suggest that p38 MAPK protects macrophages from apoptosis in atherosclerotic plaque.

Other key features of plaques that are prone to undergo disruption are collagen and elastin depletion and fibrous cap thinning (29-31). Macrophages are a source of many proteases that can degrade the extracellular matrix, and enhanced macrophage proteolytic activity induces plaque rupture (13). When lesions were analyzed for these parameters, we observed a $31 \%$ decrease in collagen content and a $40 \%$ decrease in fibrous cap thickness in the intimal area of the $p 38 a^{f l / f} \mathrm{LysMCr}^{+/-} \mathrm{Apoe}^{-/-}$lesions compared with lesions of control mice (Figure 4, A and B). Strong elastin staining was observed in the medial area, with no observable difference in staining between each group (Figure 4C). Collectively, these results show that $\mathrm{p} 38 \alpha$ deficiency in macrophages is associated with a number of markers of advanced plaque progression: enhanced macrophage apoptosis, plaque necrosis, decreased collagen levels, and decreased fibrous cap thickness.

ER stress is thought to be an important contributor to macrophage death in advanced plaque. We therefore tested whether ER stress is 
A

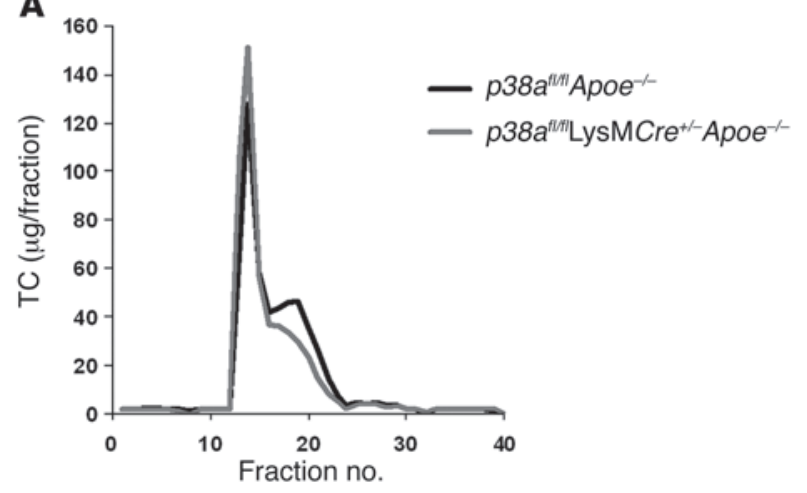

B

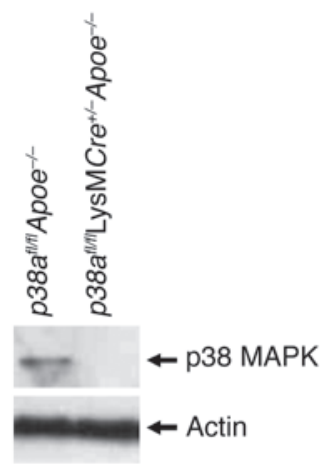

C

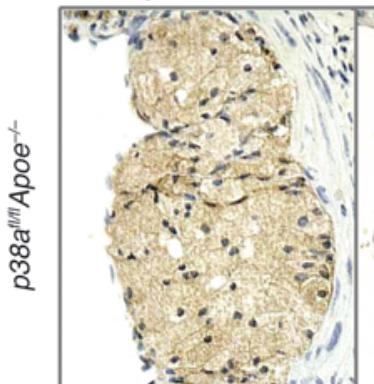

IgG control

Macrophage

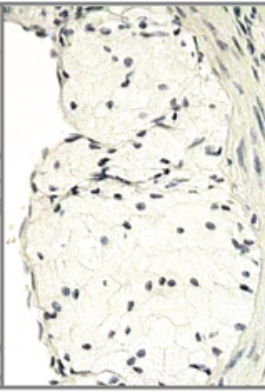

\section{0}

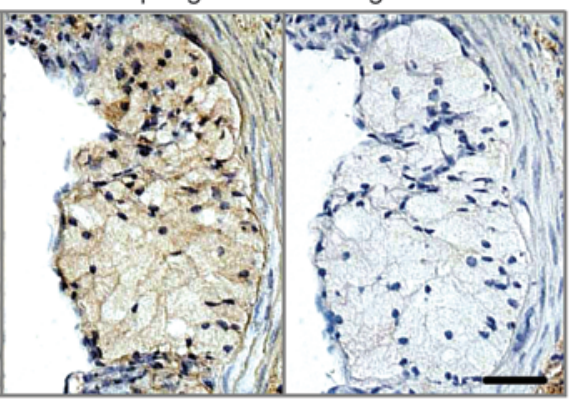

IgG control
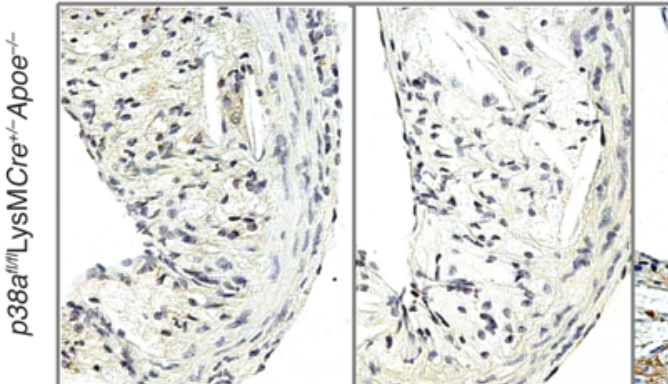

r
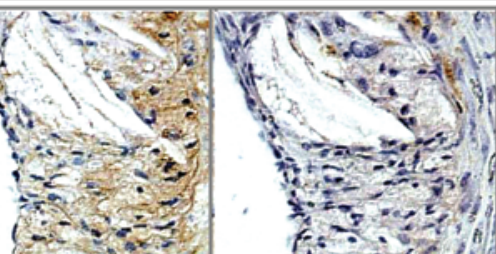

ह.
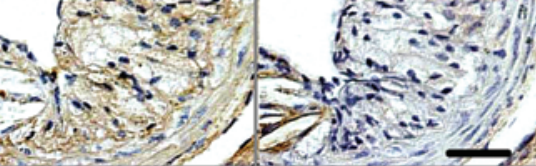

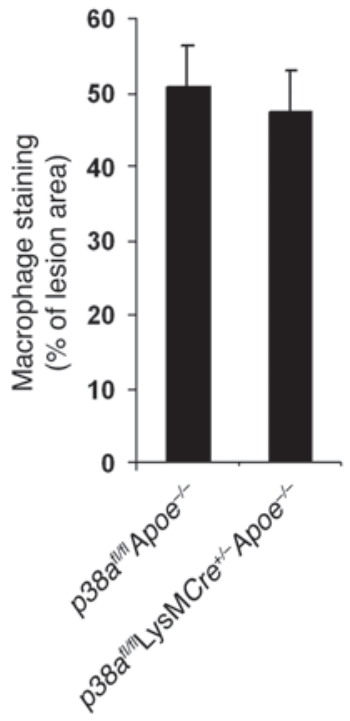

\section{Figure 1}

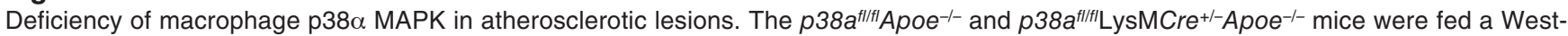
ern diet for 9 wk. (A) FPLC lipoprotein profile. TC, total cholesterol. (B) Peritoneal macrophages were isolated from $p 38 a^{f l / f I} A p o e^{-/-}$and p38a $a^{f / / f} L y s M C r e+-A p o e^{-/-}$mice. Whole cell lysates were prepared as described in Methods and immunoblotted for total p38 and actin. (C) Sections from the proximal aortas of $p 38 a^{f / / f /} \mathrm{Apoe}^{-/-}$and $p 38 \mathrm{a}^{f / / f} \mathrm{LysMCre} \mathrm{C}^{+/-} \mathrm{Apoe^{-/ }}$ mice were stained by immunohistochemistry with an antibody against phosphorylated p38 MAPK, the macrophage marker AIA31240, or the IgG control antibody. Original magnification, $\times 40$. (D) Macrophage

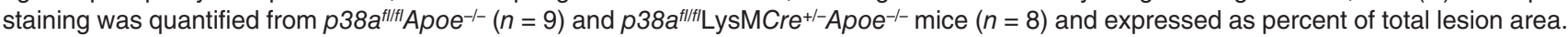

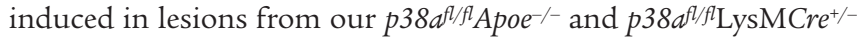
Apoe $e^{-/}$mice. As shown in Figure 5, the ER stress marker ATF3 was induced in the intimal area of both control and macrophage p38deficient lesions, which indicates that this pathway is activated in plaques of both groups of mice.

Deficiency or inhibition of $p 38 \alpha$ promotes apoptosis in ER-stressed macrophages by suppressing Akt activation. In order to determine the cause of the enhanced apoptosis observed in $p 38 a^{f l / f l} \mathrm{LysMCre}^{+/-} \mathrm{Apoe^{-/- }}$ plaques, we tested whether primary macrophages from these mice were more susceptible to ER stress-induced macrophage apoptosis. ER stress occurs in macrophages in atherosclerotic lesions and is positively correlated with increased plaque vulnerability $(14,16$, $17,21)$. As mentioned above, we previously showed that p $38 \alpha$ deficiency protects macrophages from apoptosis in a model involving accumulation of lipoprotein-derived unesterified cholesterol in the ER combined with lipoprotein-mediated PRR signaling (2).
In this model, both "hits" are required for apoptosis $(2,15,27)$. The mechanism was related to suppression of the proapoptotic ER stress effector CHOP in the setting of p38 $\alpha$ deficiency (2). These previous findings would predict that macrophage apoptosis and necrotic core formation would be suppressed in the setting of $\mathrm{p} 38 \alpha$ deficiency, the opposite of what we found in the present study.

To reconcile the difference between the previous in vitro study and our current in vivo study, we tested whether macrophage apoptosis was enhanced or inhibited by other nonlipoprotein ER stressors. Importantly, we previously found that p38 was not necessary for CHOP induction by ER stressors, such as tunicamycin or thapsigargin, but was necessary for CHOP induction by cholesterol loading (2). First, we confirmed our previous data by showing that apoptosis induced by unesterified cholesterol loading was significantly inhibited in $p 38 a^{f l / f l} \mathrm{LysMCre}^{+/-} \mathrm{Apoe^{-/- }}$ macrophages (Figure 6A). However, apoptosis induced by the 
A

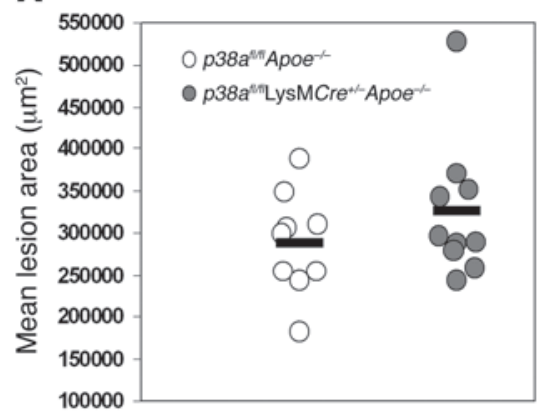

D

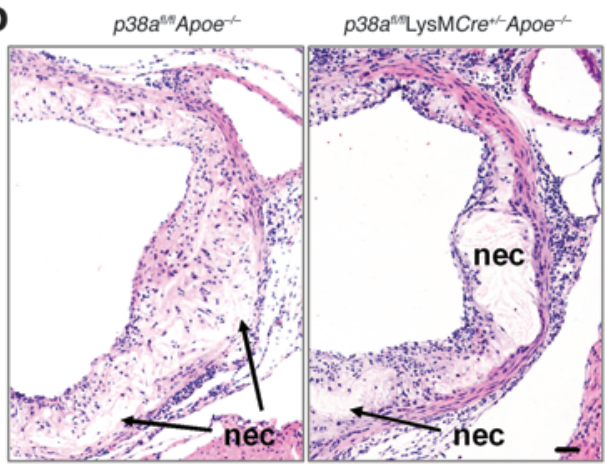

B

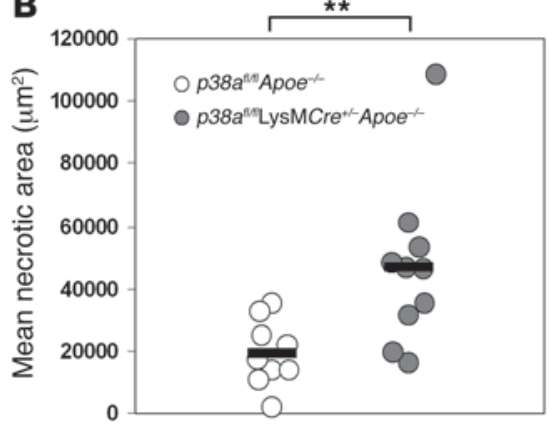

C

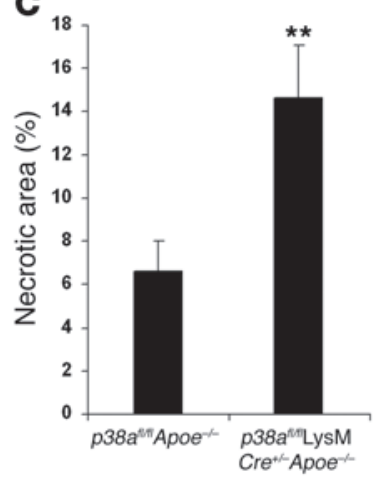

p38a" $2 y s M C r e^{+-}-$Apoe $^{--}$

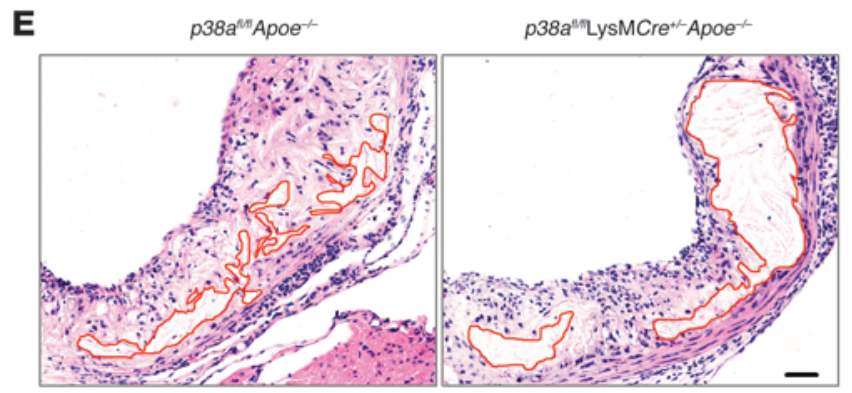

\section{Figure 2}

Deficiency in macrophage $\mathrm{p} 38 \alpha \mathrm{MAPK}$ increases lesional necrosis. (A and B) Dot plot showing the aortic root lesion area (A) and the aortic root necrotic area $(\mathbf{B})$ of individual $p 38 a^{f / / f} A_{p o e^{-/}}(n=9)$ and $p 38 a^{f / / f}$ LysMCre ${ }^{+/-} A p o e^{-/-}$mice $(n=10)$. Bars represent mean values. The difference in aortic root lesion area between groups was not significant $(P=0.462$, Mann-Whitney $U$ test). (C) Mean percent necrotic area relative to total lesion area for each group. (D) Representative images of aortic root cross sections from $p 38 a^{f / / f l} \mathrm{Apoe}^{-/-}$and $p 38 \mathrm{a}^{f / / f} \mathrm{LysMCre} \mathrm{C}^{+/-} \mathrm{Apoe^{-/- }}$ mice stained with H\&E. Necrotic areas (nec) are indicated. (E) Images from D, enlarged to demonstrate how the necrotic area was defined for quantification in each section ( $p 38 a^{f / / f l} A p o e^{-/-}, 19,018 \mu \mathrm{m}^{2} ; p 38 a^{f / / f l} L y s M C r e^{+/-} A p o e^{-/}, 45,826 \mu \mathrm{m}^{2}$ ). Red lines show the boundary of the developing necrotic core. ${ }^{* \star} P<0.01$, Mann-Whitney $U$ test. Scale bars: $50 \mu \mathrm{m}$.

ER stress inducers tunicamycin or thapsigargin was enhanced in p38afl/fl LysMCre ${ }^{+/-}$Apoe $^{-/-}$macrophages (Figure 6, A and B). ER stress-induced apoptosis was also enhanced, both in mouse peritoneal macrophages and in human monocyte-derived macrophages, when the p38 inhibitor SB202190 was used (Figure 6, C and D). Inhibition of p38 similarly enhanced macrophage apoptosis when a lower subapoptotic dose of thapsigargin, which may more closely mimic the levels of ER stress in vivo, was used (Figure $6, \mathrm{C}$ and D). Moreover, p38 inhibition further enhanced apoptosis when a PRR ligand, acetylated LDL (acetyl-LDL), was added to cells undergoing low levels of ER stress (Figure 6D). Note that in this setting, most of the cholesterol internalized from the acetylLDL was in the esterified form and did not cause ER stress, which is instead caused by the added thapsigargin $(2,18)$. We then tested whether the enhancement of apoptosis during p38 inhibition was observed with 7-ketocholesterol, an ER stress-inducing oxysterol found in oxidized LDL that is thought to promote atherogenesis (32). We found that 7-ketocholesterol-induced apoptosis was significantly enhanced under conditions of p38 inhibition (Figure $6 \mathrm{E})$. Similar results were found using serum deprivation, which has also been shown to induce an ER stress response (33). To determine whether enhanced apoptosis is a more general phenomenon of p38 inhibition, we tested 2 non-ER stress-mediated apoptotic inducers: staurosporine and UV irradiation (2). While p38 inhibition enhanced macrophage apoptosis in cells treated with stau- rosporine (Figure 6F), apoptosis was suppressed in cells exposed to UV irradiation (Figure 6G), consistent with previous reports of a proapoptotic role for p38 (34). In contrast, p38 has previously been shown to protect from pathogen-induced apoptosis $(24,35$, 36). These results suggest that the role of p38 in regulating apoptosis is both dichotomous and complex. Moreover, this complexity likely reflects the contributions of the signaling environment that influence and shape how p38 is affecting downstream signaling pathways. In the case of atherosclerosis, one of the dominant functions of p38 in macrophages is to protect cells from factors that induce apoptosis in plaque.

Previous research has shown that p38 forms a complex with MK2, Akt, and hsp27. This complex is necessary for Akt phosphorylation and activation in response to certain stimuli, such as angiotensin II stimulation, and for protecting neutrophils from apoptosis (37-40). As shown in Figure 7A, p38 was activated in response to tunicamycin and thapsigargin treatment. We therefore tested whether p38 inhibition had any effect on Akt phosphorylation in response to ER stress-inducing agents and found that SB202190 blocked Akt phosphorylation in both untreated and tunicamycin-treated macrophages (Figure 7B). Similar results were observed in p $38 \alpha$-deficient macrophages treated with thapsigargin (Figure 7C). Surprisingly, when MK2 activation was assayed, a marked decrease of phosphorylated and total MK2 protein was observed (Figure 7C), indicating that 

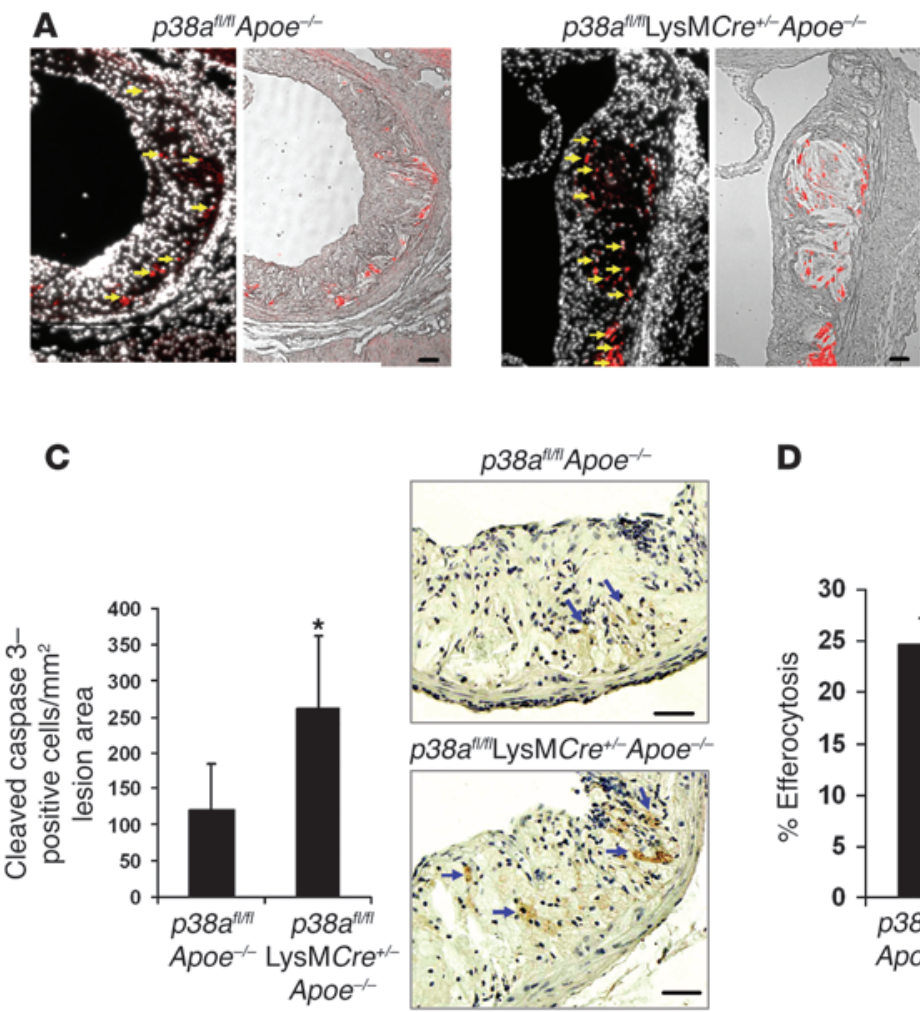

D
B

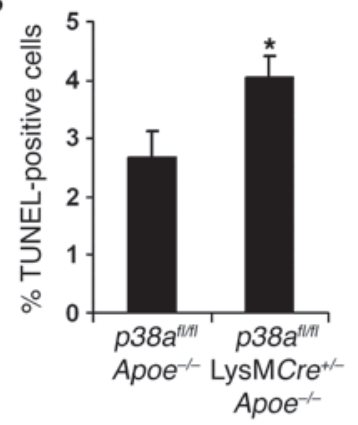

Figure 3

Increased apoptosis in plaques deficient in macrophage p38 $\alpha$ MAPK. (A-C) Sections from the proximal aortas of $p 38 a^{f / l f I} A p o e^{-/-}$and p38a fl/fliLysMCre+/-Apoe ${ }^{-/-}$mice were labeled by TUNEL to detect apoptotic cells and counterstained with DAPI to detect nuclei, or stained for activated caspase 3. (A) Shown are TUNEL-positive cells (red) in the intimal area that colocalized with DAPI-stained nuclei (arrows; left) or necrotic area (right). (B) Mean percent TUNEL- and DAPI-positive cells relative to DAPI-positive cells in the lesion ( $n=9$ per genotype). (C) Number of cleaved caspase 3-positive cells per unit of lesion area ( $n=7$ per genotype). Shown are representative images of activated caspase 3 staining in the intimal area of the lesions. Arrows indicate caspase 3-positive cells. (D) In vivo efferocytosis assay. Shown are representative images of an efferocytic event that was counted as positive. Data are expressed as the percent of F4/80-positive macrophages (red) that had ingested an apoptotic T cell (green). See Methods for details. Shown are representative images of macrophages that were counted positive for efferocytosis (arrows). Approximately $200 \mathrm{~F} 4 / 80$-positive macrophages were counted per group. ${ }^{*} P<0.05$ versus

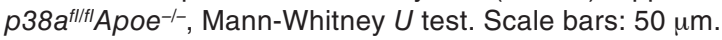

p38 is necessary for MK2 protein expression or stability. Because Akt can function in cell survival signaling $(41,42)$, we tested whether transduction of macrophages with myristoylated Akt (Myr-Akt), a constitutively active form of Akt, could inhibit ER stress-induced apoptosis under conditions of p38 inhibition. As shown in Figure 7D, transduction of macrophages with Myr-Akt increased both Akt phosphorylation and protein expression. We found that the enhancement of tunicamycin-induced apoptosis observed with SB202190 or in p38 $\alpha$-deficient macrophages was completely suppressed by Myr-Akt (Figure 7, E and F). Myr-Akt offered no additional protection from macrophage apoptosis induced by tunicamycin or thapsigargin alone. We next tested the effects of combining p38 and Akt inhibitors. If p38 inhibitors work synergistically with Akt inhibitors to enhance ER stress-induced apoptosis, then this would suggest that p38 may have Akt-independent effects that enhance macrophage death. We found that inhibition of Akt with the inhibitor LY294002 markedly augmented apoptosis during ER stress (Figure 7G). However, no synergism was observed with the combination of LY294002 and SB202190, which suggests that p38 and Akt act through the same pathway to suppress macrophage apoptosis during ER stress. Thus, it appears that activation of p38 MAPK during the ER stress response leads to phosphorylation of Akt, which has antiapoptotic consequences.

We next tested whether levels of phosphorylated Akt are

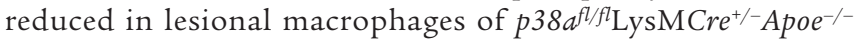
mice, as they were in cultured p $38 \alpha$-deficient macrophages. Using anti-phospho-Akt immunohistochemistry, we found that Akt was phosphorylated in both the intima and the media of $p 38 a^{f l / f l} \mathrm{Apoe}^{-/-}$control lesions (Figure 8A). Akt has previously been shown to function in both cytoplasmic and nuclear compartments $(43,44)$. While lesions from $p 38 a^{f l / f l} \mathrm{LysMCre}^{+/-} \mathrm{Apoe} \mathrm{e}^{-/-}$ mice also had numerous immunopositive cells in the media, there were fewer of these cells in the intima compared with control lesions (Figure 8A, arrows). Quantification of these observations revealed a $64 \%$ reduction in immunopositive nuclei within the intimal area (Figure 8B). We further characterized these lesions by testing whether phosphorylated Akt colocalized with macrophages in the intimal area of the lesion. As shown in Figure 8C, Akt phosphorylation was observed within and around the DAPI-stained nuclei and was markedly reduced in the p38aflfll LysMCre ${ }^{+/-}$Apoe $e^{-/-}$lesions. The phosphorylated Akt over- 
A $p 38 a^{f t / t} \mathrm{Apoe}^{-\alpha}$

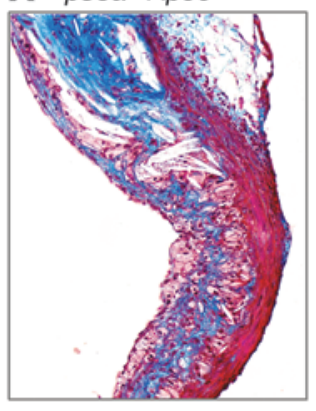
p38a $a^{\text {th/7}}$ LysMCre $^{+-} \mathrm{Apoe}^{-1-}$

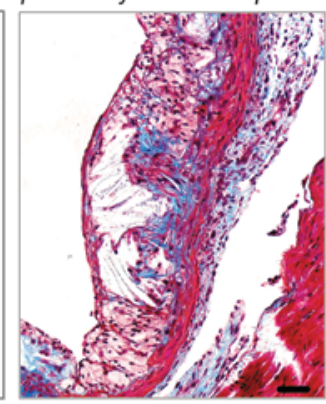

B

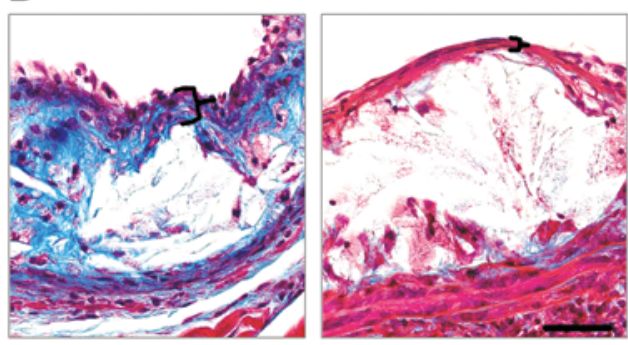

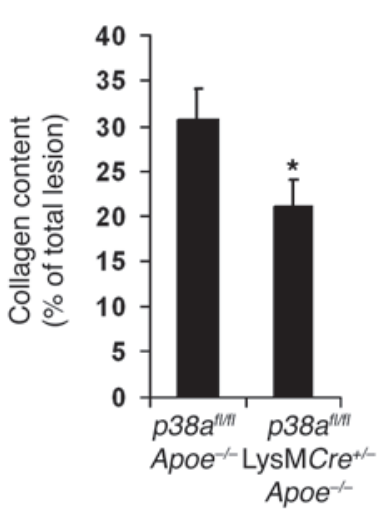

C

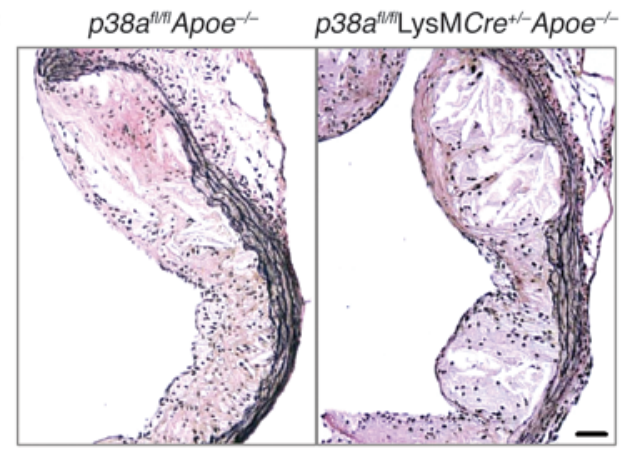

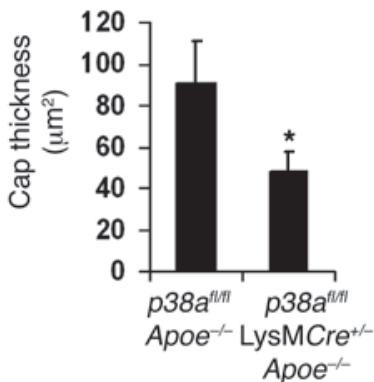

Figure 4

Fibrous cap thinning and collagen depletion in plaques deficient in macrophage p38 $\alpha$ MAPK. (A-C) Masson trichrome stain for collagen (blue) and elastin in $p 38 a^{f / / t} \mathrm{Apoe}^{-/-}$and $p 38 a^{f / / f} \mathrm{~L}$ ysMCre ${ }^{+/-} \mathrm{Apoe}^{-/-}$lesions. (A) Quantified data are expressed as the average percent collagen relative to total lesion area ( $n=9$ per genotype). (B) Fibrous cap thickness was measured and quantified. Mean values of data from 9 mice are shown. Bracketed regions show a representative measurement of the fibrous and afibrous cap in the $p 38 a^{f / / t} A p o e^{-/-}$and $p 38 a^{f / / f} L y s M C r e^{+/-} A p o e^{-/-}$mice, respectively. (C) Elastin (black) in the tunica media. ${ }^{*} P<0.05$ versus $p 38 a^{f / / t} A p o e^{-/-}$, Mann-Whitney $U$ test. Scale bars: $50 \mu \mathrm{m}$.

lapped with areas positive for macrophages. However, there were areas of macrophage staining that did not overlap with Akt phosphorylation. These data suggest that not all macrophages in the intima have equally high levels of activated Akt. We conclude that p38 $\alpha$ deficiency leads to reduced Akt activation in macrophagerich intimal regions of atherosclerotic plaques.

\section{Discussion}

Several previous studies have shown that activation of p38 MAPK can have pro- or antiapoptotic effects depending on the cellular environment $(2,23,24,34-36)$. The important finding in the present study is that in advanced atherosclerotic plaque, p38 $\alpha$ plays a prosurvival role in macrophages. In cell culture, free cholesterol-induced macrophage apoptosis, but not tunicamycin- or thapsigargin-induced apoptosis, is suppressed under conditions of p38 inhibition (2). This earlier observation correlated with the necessity of $\mathrm{p} 38$ for the induction of the proapoptotic UPR effector CHOP: with cholesterol loading, p38 was needed for CHOP induction, while with other ER stressors, CHOP induction did not depend on p38 (2). Thus, the role of p38 in apoptosis may depend on the balance between a 38 -CHOP proapoptotic pathway and a p38-Akt prosurvival pathway. Free cholesterol loading may somehow promote the $\mathrm{p} 38-\mathrm{CHOP}$ pathway and/or suppress the p38-Akt pathway. As to why the net result of macrophage p38 $\alpha$ in advanced Apoe $e^{-/-}$lesions is prosurvival, many other noncholesterol ER stressors exist in lesions that may promote apoptosis, such as oxidant stress and peroxynitrite (45), insulin resistance (46), glucosamine (47), saturated fatty acids (48), hypoxia
(49), homocysteine (50), oxidized phospholipids (14), oxysterols such as 7 -ketocholesterol (16), and growth factor withdrawal (33). There is evidence that macrophage cholesterol loading does occur in advanced plaques and is proapoptotic $(18,19,51)$. Thus, an alternative explanation is that the buildup of these additional factors could trump the p38-CHOP pathway that is induced by free cholesterol loading by enhancing the p38-Akt pathway.

The finding that inhibition or absence of p38 triggered apoptosis in cells exposed to subapoptotic doses of ER stressors fits well with a fundamental concept we developed regarding ER stressinduced apoptosis. This concept posits that prolonged ER stress in vivo is unlikely to be at a level that would induce apoptosis by itself, but that cell death would be triggered when ER stress was combined with one or more additional noxious hits. This concept is supported by the findings of our previous in vitro and in vivo studies that deficiency of the ER stress signaling pathways or PRR signaling (CD36 and type A scavenger receptor) decreases macrophage apoptosis and plaque necrosis $(15,19,52)$. Examples of proapoptotic second hits that can occur in vivo include PRR ligands, such as those engaging the type A scavenger receptor CD36 and TLR4, or additional ER stressors such as insulin resistance (discussed below), 7-ketocholesterol, and serum deprivation (our unpublished observations and refs. 2, 27, 32, 46). Based on our present results with staurosporine, there may well be other unidentified factors that could also enhance macrophage apoptosis in plaque under conditions of p 38 deficiency. These second hits exert their effects by enhancing ER stress-induced proapoptotic processes or suppressing ER stress-induced cell survival sig- 


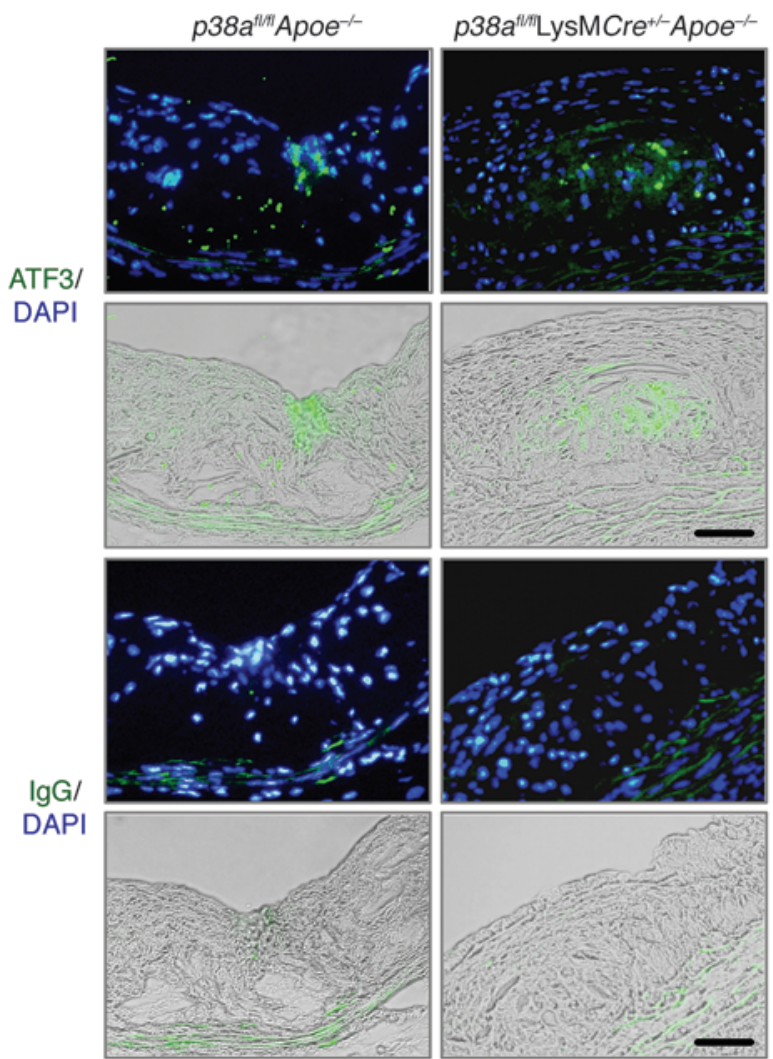

naling (20). For example, ER stress-induced macrophage apoptosis is dependent on a set of proapoptotic events such as CHOP induction, STAT1 phosphorylation, and JNK activation $(2,15,18$, $20,27)$. When these proapoptotic events are activated, they are counterbalanced by survival pathways (20). Because p38 $\alpha$ deficiency accelerates ER stress-induced apoptosis by inhibiting Akt phosphorylation, Akt inhibition may be considered as another example of a second hit in this model. Therefore, the loss of this important survival pathway tips the survival-death pathway in favor of apoptosis (Figure 9). Consistent with this hypothesis, a recent study demonstrated that deficiency of Akt 1 increased macrophage and endothelial cell apoptosis (53). However, it was also noticed that lesion size increased. This phenotype appears to be caused by the lack of AKT expression in nonhematopoietic cells because the effect on lesion size was lost in wild-type mice transplanted with $A k t 1^{-1-}$ bone marrow (53).

Macrophages lacking insulin receptors, as a model of macrophage insulin resistance, have enhanced ER stress-induced apoptosis (46). Most importantly, when lethally irradiated $\mathrm{Ldlr}^{-/-}$mice were reconstituted with these macrophages and then fed an atherogenic diet, advanced lesional macrophage apoptosis and necrotic core formation were increased (46). We have found that the p38 inhibitor markedly suppressed Akt activation and further enhanced ER stress-induced apoptosis in $\mathrm{Insr}^{-1} \mathrm{Ldlr}^{/-}$macrophages in vitro (our unpublished observations). Thus, the combination of insulin resistance and p38 inhibition in macrophages synergistically enhance the ER stress apoptosis pathway through Akt suppression.

The mechanism of the p38-Akt link shown here may involve a p38-MK2-Akt pathway. MK2 exists in a complex with p38, hsp27, and Akt, leading to p38-mediated phosphorylation of

\section{Figure 5}

Induction of ER stress in vivo. Aortic sections from $p 38 a^{f / f l} A p o e^{-/-}$and p38a fl/fliLysMCre ${ }^{+/-} \mathrm{Apoe}^{-/-}$mice were stained by immunofluorescence with an antibody against ATF3 or an IgG control antibody. ATF3-positive cells (green) in the intimal area (green staining overlaid on bright field) colocalized with and around the DAPI-stained nuclei (green staining overlaid on DAPI). Scale bars: $50 \mu \mathrm{m}$.

MK2 and subsequent MK2-mediated phosphorylation of Akt on $\operatorname{Ser}^{473}(38,40)$. Interestingly, MK2 protein levels were suppressed by p38 deficiency. These data suggest that p38 activation may somehow control MK2 protein expression or stability. Previous data from our lab have shown that MK2 deficiency leads to a similar loss of $\mathrm{p} 38$ protein levels and may be related to the ability of MK2 to stabilize p38 $(2,54)$. The p38-MK2 interaction may therefore be codependent on the stabilities of both proteins. With regard to regulation of Akt phosphorylation, inhibition of p38 has previously been shown to suppress MK2-mediated Akt phosphorylation in angiotensin II-treated smooth muscle cells (39). Moreover, MK2 and hsp27 were recently shown to regulate Akt activation and neutrophil apoptosis by mediating a MK2-Akt interaction $(38,40)$. Therefore, the lack of MK2-mediated Akt activation may explain why accelerated macrophage apoptosis occurs during p38 inhibition in vivo. Of interest in this regard, holodeficiency of MK2 in mice suppresses macrophage recruitment and foam cell formation during atherosclerosis (3). Our present findings with macrophage-specific knockout of p38 suggest that the effect of MK2 in the holoknockout may be mediated by other cell types involved in lesional macrophage recruitment, such as endothelial cells.

Inhibitors to p38 are currently being tested in clinical trials as antiinflammatory drugs for rheumatoid arthritis, COPD, atherosclerosis, and Crohn disease (1). In a vascular injury model, inhibition of p38 MAPK specifically in smooth muscle cells reduced neointimal formation, suggesting a beneficial role for the use of these inhibitors in angioplasty or stent delivery (4). However, the role of p38 inhibition in other cell types of neointimal formation was not tested in that study. While other studies have shown that macrophage deletion of p38 has marked effects of reducing inflammation in murine models of skin injury and septic shock $(8,9)$, here we found no reduction in lesion size or macrophage content. Consistent with our results, atherosclerotic lesion size and macrophage content were also unaffected when a p38 inhibitor was given to Apoe $e^{-/-}$mice infused with angiotensin II (55). Apoptosis or other features of advanced plaque morphology, such as necrotic area, were not measured in that study. Holodeficiency of MK2 reduced plaque size and macrophage content (3), but again, effects on plaque necrosis were not reported. The in vivo observations and mechanistic studies presented here raise the possibility that p38 inhibitors may enhance macrophage death and necrotic core formation. Thus, further studies are needed to determine the net effect of p38 inhibitors on features of advanced plaque morphology and subsequent acute coronary events.

\section{Methods}

Reagents. Falcon tissue culture plastic was purchased from Fisher Scientific. Tissue culture media, cell culture reagents, and heat-inactivated FBS were purchased from Invitrogen. The ACAT inhibitor Sandoz 58-035 (3-[decyldimethylsilyl]-N-[2-(4-methylphenyl)-1-phenylethyl]propanamide; 

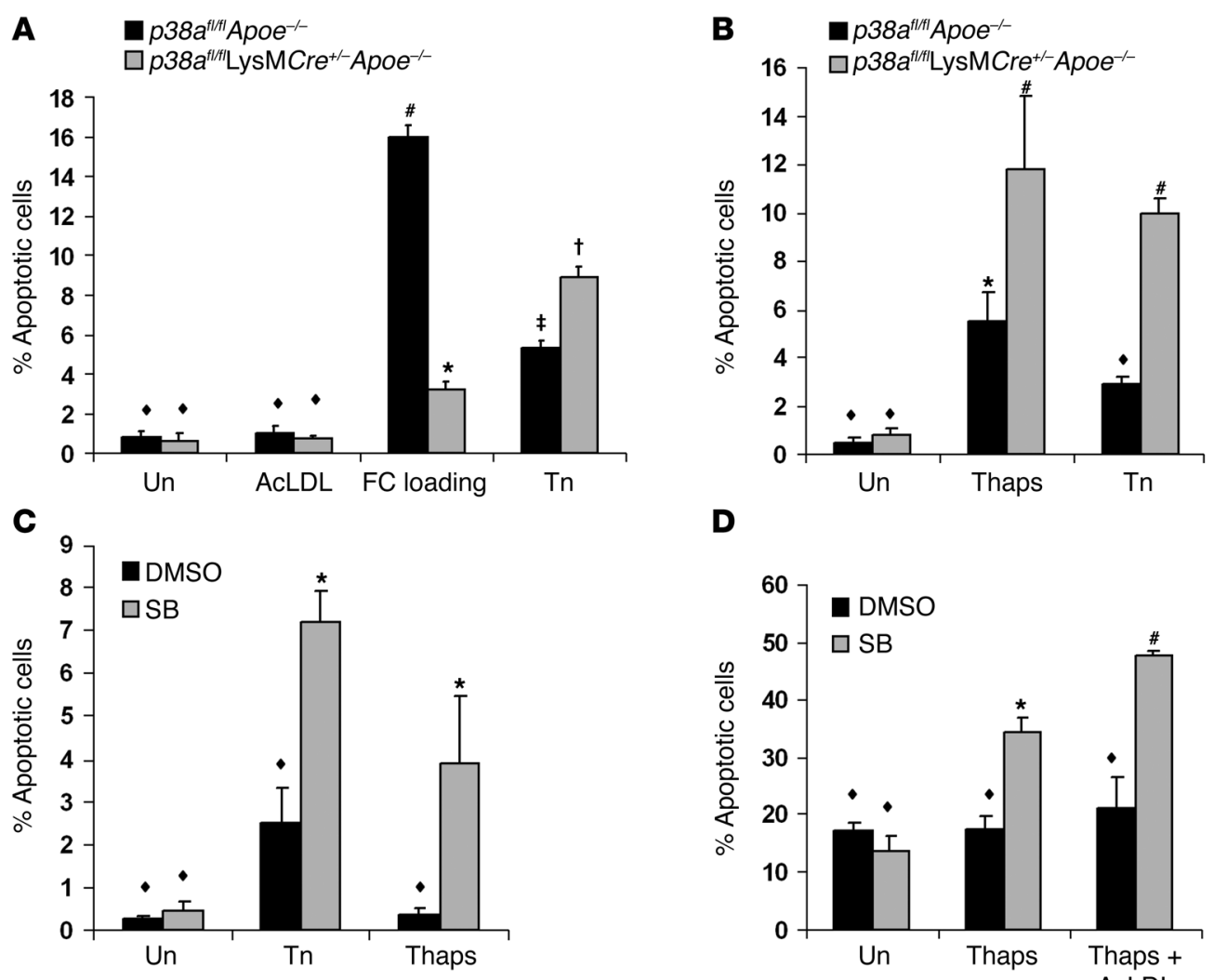

D
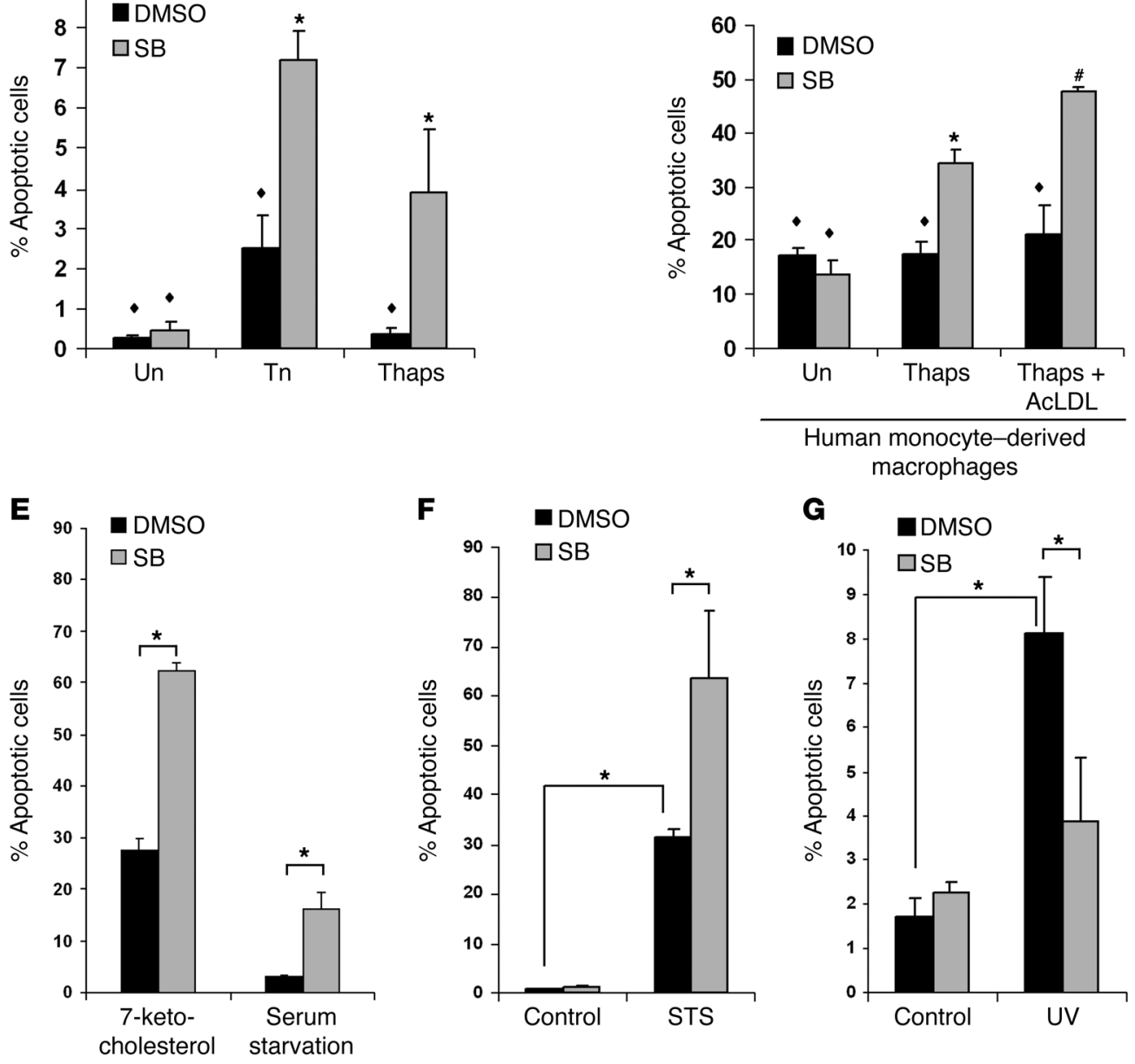

Figure 6

Inhibition of p38 $\alpha$ MAPK accelerates macrophage apoptosis during ER stress. (A) p38a $a^{f / / f} A p o e^{-/-}$and $p 38 a^{f / / f} L y s M C r e e^{+/-A p o e^{-/-}}$peritoneal macrophages were left untreated (Un), treated with acetyl-LDL (AcLDL), cholesterol-loaded (acetyl-LDL plus 58035; FC loading) for 16-18 h, or treated with $5 \mu \mathrm{g} / \mathrm{ml}$ tunicamycin ( $\mathrm{Tn}$ ) for $24 \mathrm{~h}$, after which cells were assayed for apoptosis. Data are expressed as the percent of total cells that stained with annexin V and propidium iodide. (B) Cells as in A were untreated or treated with $2 \mu \mathrm{M}$ thapsigargin (Thaps) or $5 \mu \mathrm{g} / \mathrm{ml}$ tunicamycin for $24 \mathrm{~h}$, then assayed as in $\mathbf{A}$. ( $\mathbf{C}$ and $\mathbf{D}$ ) Wild-type mouse peritoneal (C) or human monocyte-derived (D) macrophages were pretreated with $10 \mu \mathrm{M}$ SB202190 (SB) or the vehicle DMSO control for $1 \mathrm{~h}$ and then treated with $5 \mu \mathrm{g} / \mathrm{ml}$ tunicamycin or $0.25 \mu \mathrm{M}$ thapsigargin (C) or with $0.25 \mu \mathrm{M}$ thapsigargin or $0.25 \mu \mathrm{M}$ thapsigargin plus acetyl-LDL (D) for $24 \mathrm{~h}$ and assayed for apoptosis as described in $\mathbf{A}$. In $\mathbf{A}-\mathbf{D}$, common symbols denote differences that are not statistically significant $(P>0.05)$, while different symbols denote statistically significant differences $(P<0.05)$; ANOVA with Student-Newman-Keuls post-test. (E-G) Peritoneal macrophages were pretreated for $1 \mathrm{~h}$ with $10 \mu \mathrm{M}$ SB202190 or the vehicle DMSO control. Cells were then given $20 \mu \mathrm{g} / \mathrm{ml}$ 7-ketocholesterol or serum starved for $18 \mathrm{~h}(\mathbf{E})$, treated with $100 \mathrm{nM}$ staurosporine (STS) for $24 \mathrm{~h}(\mathbf{F})$, or UV irradiated and followed for $7 \mathrm{~h}(\mathbf{G})$. Cells were then assayed for apoptosis as described in A. All data are mean \pm SEM $(n=4)$. In $\mathbf{E}-\mathbf{G}$, ${ }^{*} P<0.05$, ANOVA with Student-Newman-Keuls post-test. 

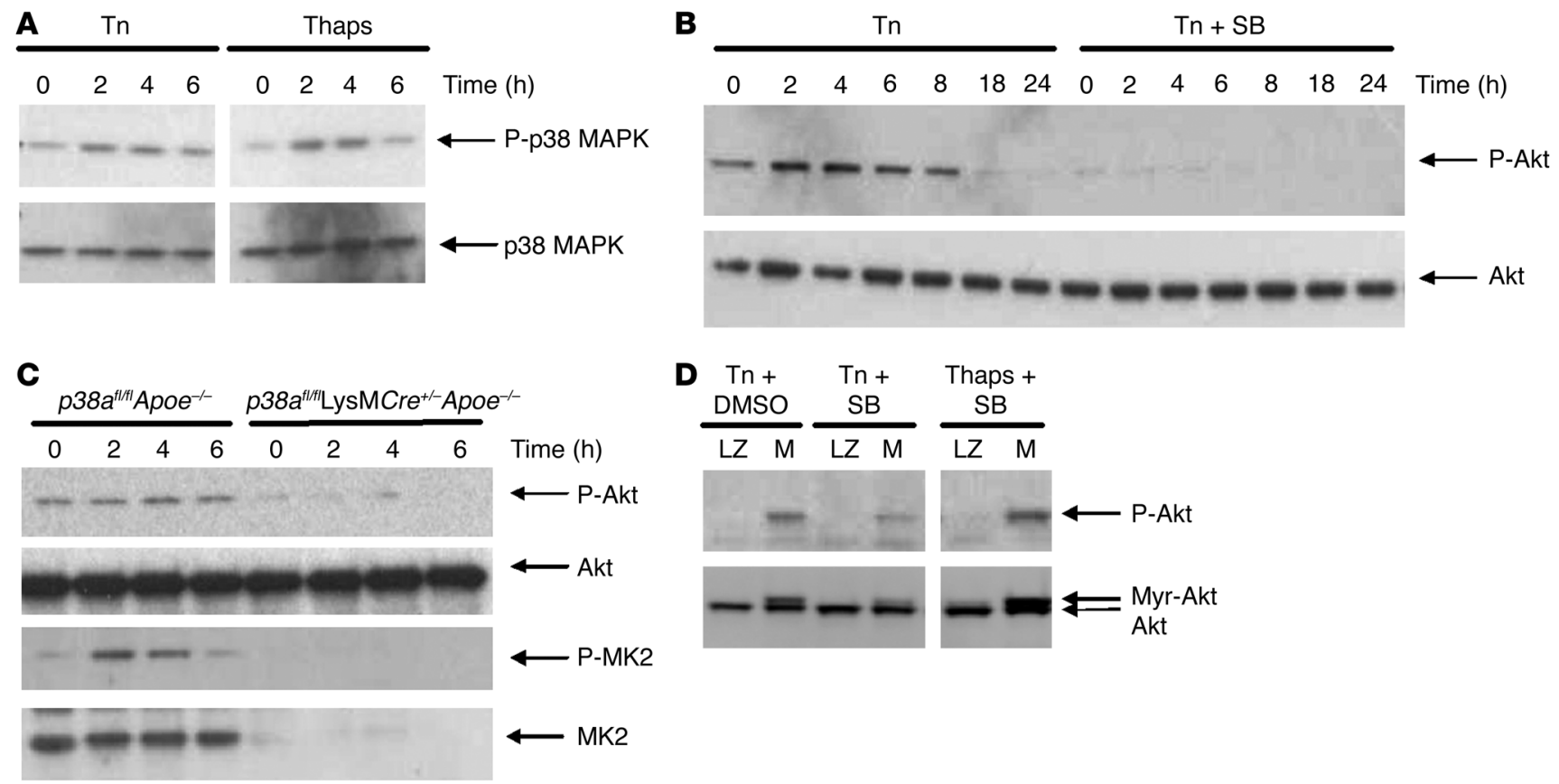

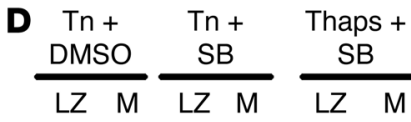

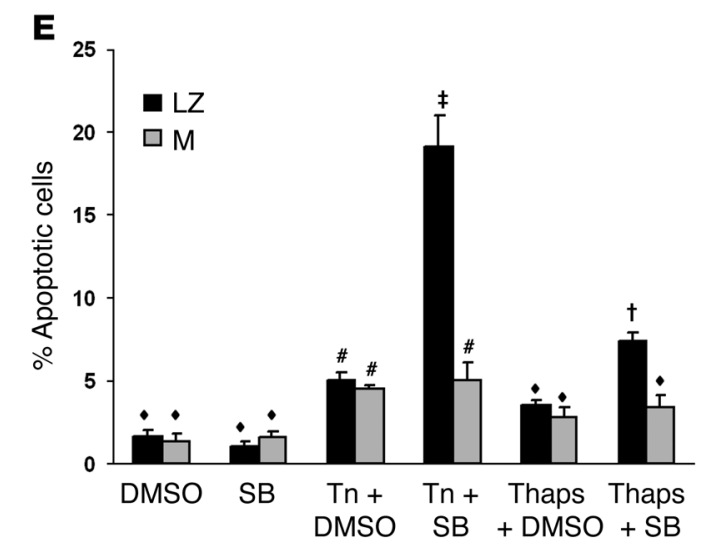

$\mathbf{F}$
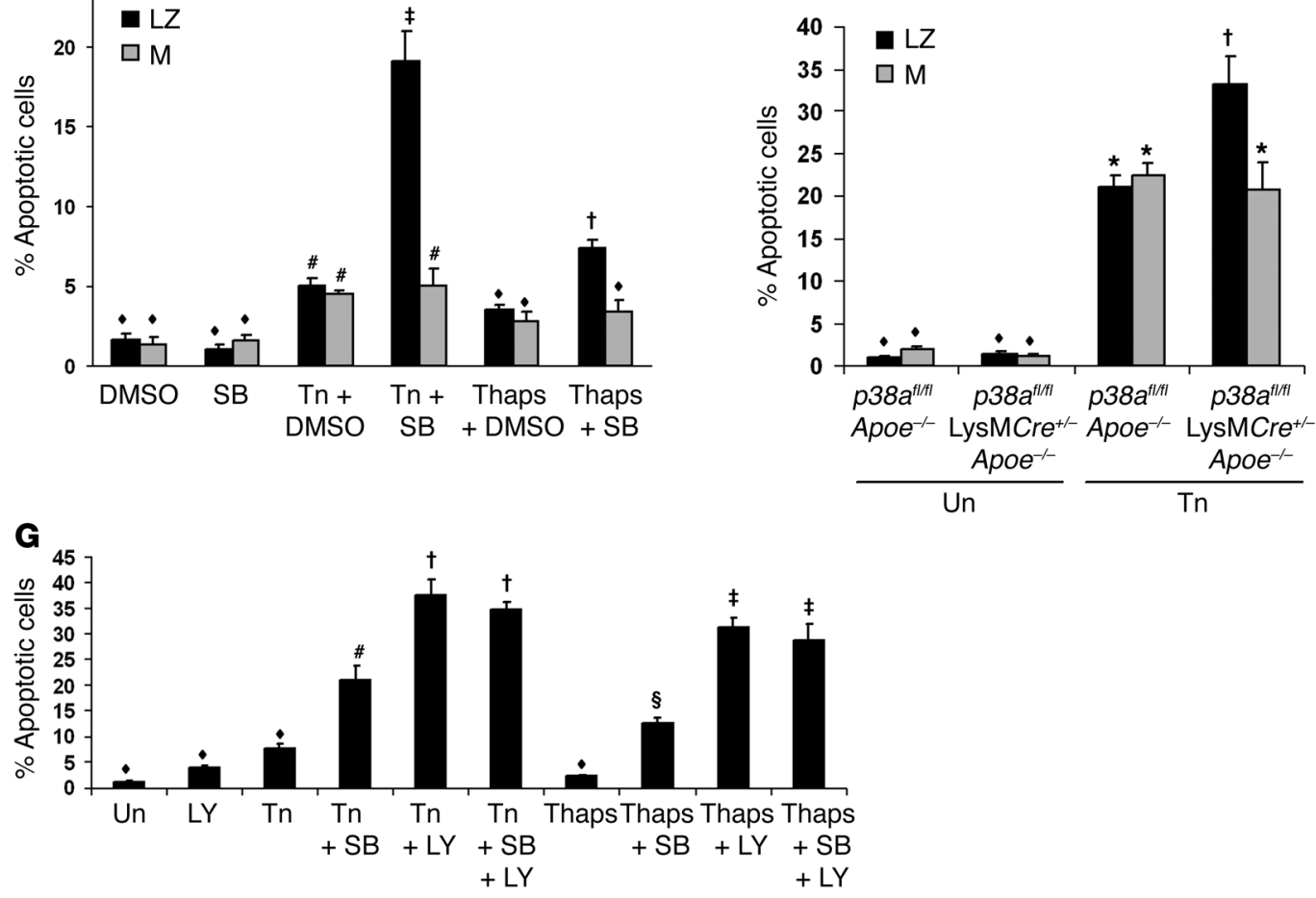


\section{Figure 7}

p38 $\alpha$ MAPK inhibition accelerates ER stress-induced macrophage apoptosis by suppressing Akt. (A) Wild-type peritoneal macrophages were treated with $5 \mu \mathrm{g} / \mathrm{ml}$ tunicamycin or $0.25 \mu \mathrm{M}$ thapsigargin for the indicated times. Lysates were immunoblotted for activated phosphorylated Thr180/Tyr ${ }^{182}$-p38 MAPK (P-p38) and total p38. (B) Peritoneal macrophages were pretreated with $10 \mu \mathrm{M}$ SB202190 or DMSO control for $1 \mathrm{~h}$ and then treated with tunicamycin for the indicated times. Lysates were immunoblotted for activated phosphorylated Ser ${ }^{473}$-Akt (P-Akt) and total Akt. (C) Bone marrow-derived macrophages were treated with $0.25 \mu \mathrm{M}$ thapsigargin for the indicated times. Lysates were immunoblotted for activated phosphorylated Ser473-Akt, activated phosphorylated Thr ${ }^{334}$-MK2 (P-MK2), and total Akt or MK2. (D) Peritoneal macrophages were transduced with adenovirus containing constitutively active MyrAkt (M) or control LacZ (LZ) at $500 \mathrm{MOI}$ for $18 \mathrm{~h}$. After the infection, macrophages were pretreated with $10 \mu \mathrm{M}$ SB202190 or the vehicle DMSO control followed by tunicamycin or thapsigargin treatment for $24 \mathrm{~h}$. Lysates were immunoblotted for phosphorylated Akt and total Akt. Myr-Akt is shown as a shift in mobility. (E) Peritoneal macrophages were transduced with adenovirus and treated as in $\mathbf{D}$ and then measured for apoptosis. (F) Bone marrow-derived macrophages were transduced, treated with tunicamycin for $24 \mathrm{~h}$, and measured for apoptosis. (G) Peritoneal macrophages were pretreated with $10 \mu \mathrm{M}$ LY294002 (LY) or SB202190 alone or in combination followed by no treatment, tunicamycin, or thapsigargin for $24 \mathrm{~h}$, and measured for apoptosis. Common symbols denote differences that are not statistically significant $(P>0.05)$, while different symbols denote statistically significant differences $(P<0.05)$; ANOVA with Student-Newman-Keuls post-test.

ref. 56) was from Sigma-Aldrich; a $10-\mathrm{mg} / \mathrm{ml}$ stock was made in DMSO and used at a concentration of $10 \mathrm{mg} / \mathrm{ml}$. All other chemical reagents, including tunicamycin, thapsigargin, concanavalin A, SB202190, LY294002, 7-ketocholesterol, and DAPI, were purchased from SigmaAldrich. The 7-ketocholesterol was dissolved in $100 \%$ ethanol at $10 \mathrm{mg} / \mathrm{ml}$, vortexed extensively, and passed through a $0.22-\mu \mathrm{m}$ filter. Ethanol alone was used as the vehicle control. For serum starvation, the growth medium was removed and replaced with DMEM plus 1\% penicillin/streptomycin/ glutamine for 18-24 h. Antibodies against phosphorylated p38 MAPK, p38 MAPK, phosphorylated Akt, Akt, phosphorylated MK2, MK2, and cleaved caspase 3 for Western blotting and immunocytochemistry were purchased from Cell Signaling Technology. The rabbit polyclonal antibody to AIA31240 was purchased from Accurate Chemical and Scientific Corporation, and PE-conjugated anti-F4/80 was purchased from Cedarlane. The mouse monoclonal antibody to actin was purchased from Chemicon. The normal rabbit IgG control antibody was purchased from R\&D Systems. The horseradish peroxidase-conjugated donkey antimouse and donkey anti-rabbit IgG secondary antibodies were purchased from Jackson Immunoresearch Laboratories. Donkey anti-rabbit Alexa Fluor 488- and Alexa Fluor 564-conjugated secondary antibodies were purchased from Invitrogen. The Vybrant Annexin V/Propidium Iodide Apoptosis Assay kit no. 2 was from Invitrogen. LDL (d, 1.020-1.063 g/ml) from fresh human plasma (obtained from New York Blood Center) was isolated by ultracentrifugation (57). Acetyl-LDL was prepared from a reaction with acetic anhydride, as described previously (58), and used at a concentration of $50 \mu \mathrm{g} / \mathrm{ml}$ in all experiments.

Mice. Macrophages deficient in $\mathrm{p} 38$ were obtained from $p 38 a^{f / f l}$ mice crossed with LysMCre; C57BL/6 mice $(59,60)$. These mice were then crossed onto $A p o e^{-/-}$mice. Mice used for the atherosclerosis study were generated from $p 38 a^{\text {fl/fl }} \mathrm{LysMCr} e^{+/-} A p o e^{-/-}$and $p 38 a^{f / f l} \mathrm{Apoe}^{-/-}$(no Cre) parents, which had been backcrossed into the C57BL/6J background for more than 8 generations. At $8 \mathrm{wk}$ of age, female mice were placed on a Western-type diet (TD88137; Harlan Teklad) for $9 \mathrm{wk}$. All animal procedures used in this study were approved by the Institutional Animal Care and Use Committee of Columbia University.

Plasma cholesterol and triglyceride measurements. Plasma was collected from mice, after $12 \mathrm{~h}$ of fasting, via exsanguination from left-ventricular puncture. Total plasma cholesterol and triglyceride levels were measured using commercially available kits (Wako Pure Chemical Industries). Pooled plasma from 3 mice was used to obtain lipoprotein profiles. Profiles were obtained using FPLC gel filtration and a Superose 6 column (Amersham Pharmacia) at a flow rate of $0.2 \mathrm{ml} / \mathrm{min}$, followed by cholesterol assays of the fractions. Plasma from 2-3 mice was pooled to obtain the FPLC profile data.

Atherosclerotic lesion analysis. For morphometric lesion analysis, sections were stained with Harris $\mathrm{H} \& \mathrm{E}$, and analysis was performed by 2 independent observers blinded to genotype. Total intimal lesion area (from internal elastic lamina to the lumen) and acellular/anuclear areas (negative for hematoxylin-positive nuclei) per cross section were quantified by taking the average of 6 sections spaced $30 \mu \mathrm{m}$ apart beginning at the base of the aortic root. The necrotic core was defined as a clear area that was H\&E free. Boundary lines were drawn around these regions, and the area measurements were obtained by image analysis software (see below). A $3,000-\mu \mathrm{m}^{2}$ threshold was implemented in order to avoid counting very small clear areas frequently observed in H\&E-stained sections that likely do not represent substantial areas of necrosis. Using this method, a $97 \%$ agreement in the percent necrotic area was calculated between our 2 independent observers. Morphological analysis of collagen was performed with Masson trichrome stain (Richard-Allan Scientific) and elastin stain (hematoxylin-iodine-ferric chloride; Sigma-Aldrich) on duplicate sections taken about 60-70 $\mu \mathrm{m}$ from the base of the aortic root. Images were viewed and captured with a Nikon Labophot 2 microscope equipped with an Olympus DP25 color digital camera attached to a computerized imaging system with Image-Pro-Plus software (version 3.0; Mediacybernetics). Fibrous cap thickness was quantified by choosing the largest necrotic core from duplicate sections and taking a measurement from the thinnest part of the cap, determined by measuring the area between the outer edge of the cap and the necrotic core boundary.

Antibody staining of mouse sections. Mouse sections were deparaffinized in xylene and hydrated in water. Antigen retrieval was obtained by maintaining slides for $20 \mathrm{~min}$ in sub-boiling sodium citrate buffer $(10 \mathrm{mM}$ sodium citrate in distilled water, $\mathrm{pH}$ 6.0) for AIA31240, p38, and ATF3 staining or in 1 mM EDTA ( $\mathrm{pH}$ 8.0) for phosphorylated Akt and cleaved caspase 3 . The sections were incubated with the appropriate antibody overnight and stained for immunofluorescence of immunohistochemistry according to the protocols provided by Cell Signaling Technology. For immunohistochemistry, the sections were then treated with $3 \% \mathrm{H}_{2} \mathrm{O}_{2}$ to inactivate the endogenous peroxidase. The sections were stained using the rabbit $\mathrm{ABC}$ staining system according to the manufacturer's protocol (Santa Cruz Biotechnology Inc.). The sections were then counterstained with Mayer's H\&E and examined by light microscopy.

Isolation of mouse peritoneal macrophages. Peritoneal macrophages from adult female C57BL/6J mice and all mutant mice used in this study were harvested $3 \mathrm{~d}$ after i.p. injection of concanavalin A or $4 \mathrm{~d}$ after i.p. injection of methyl-BSA in mice previously immunized with this antigen (2, 61). Macrophages were harvested $4 \mathrm{~d}$ later by peritoneal lavage. All macrophages were grown in full medium containing DMEM (25 mM glucose), $10 \%$ FBS, 20\% L cell-conditioned medium, and 1\% penicillin/streptomycin/glutamine. The medium was replaced every $24 \mathrm{~h}$ until cells reached $90 \%$ confluency. On the day of the experiment, the cells were washed 3 times in warm PBS and incubated as described in the figure legends.

Isolation of human monocyte-derived macrophages. Human monocytederived macrophages were obtained from 1 unit of buffy coat (New York 
A $\lg G$

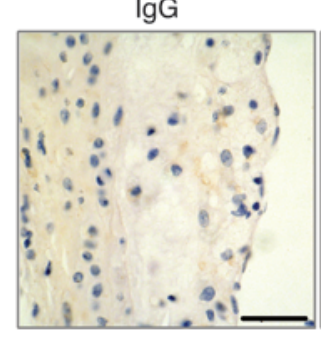

P-Akt
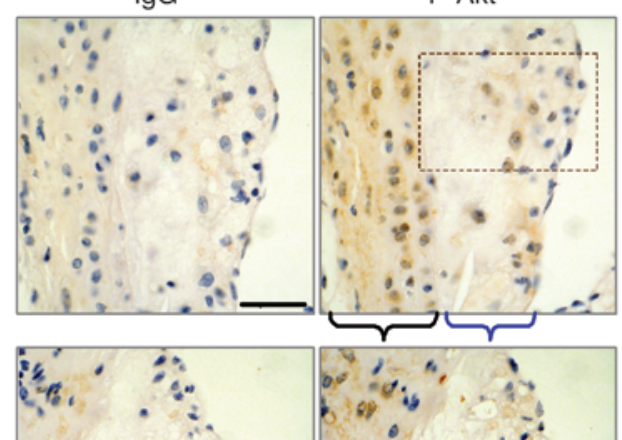

p38a $a^{f / 7}$

LysMCre+t/-

$\mathrm{Apoe}^{-/}$

C
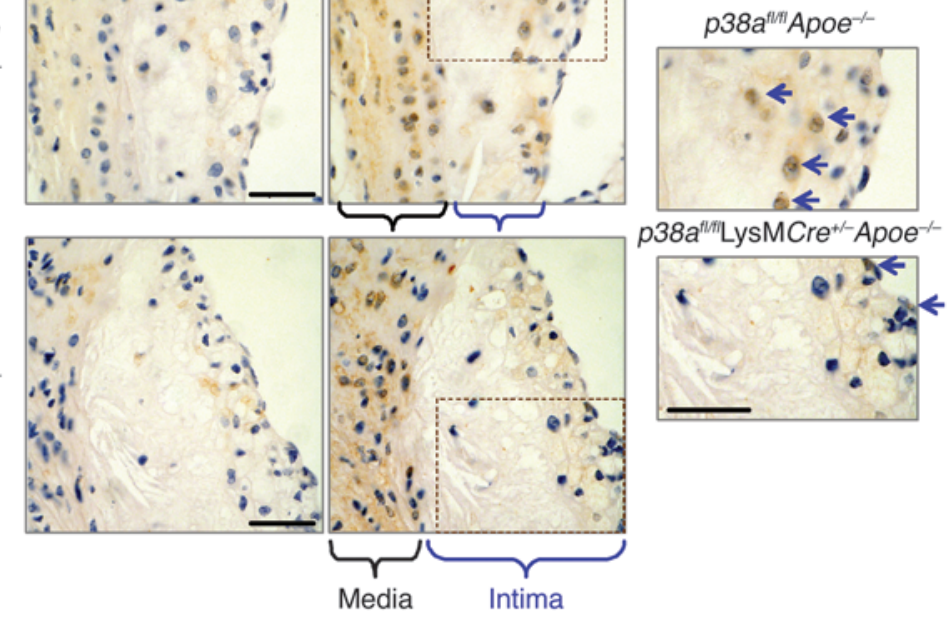

Macrophage AIA/DAPI

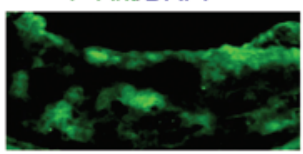

p38atitt

Apoe $^{-/}$
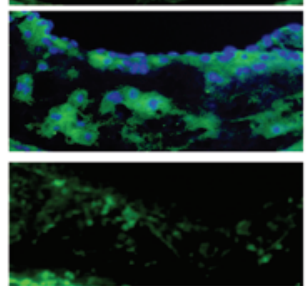

p38a $a^{n t m}$

LysMCre+/-

$\mathrm{Apoe}^{-/}$
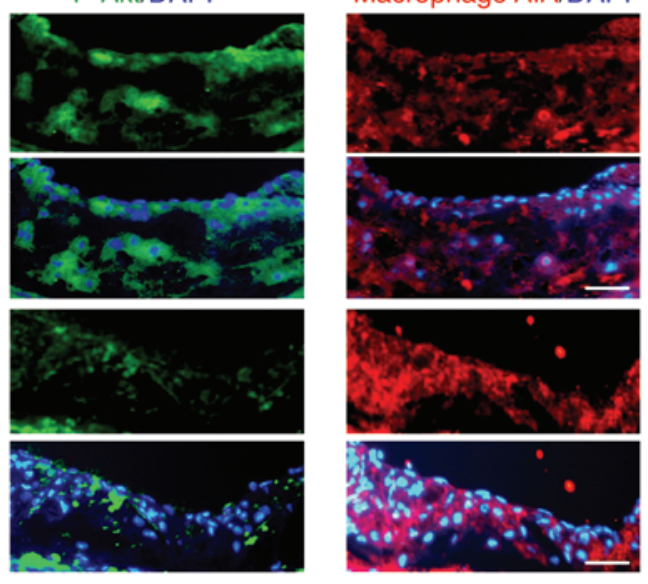

\section{Figure 8}

Akt phosphorylation is suppressed by macrophage p38 $\alpha$ deficiency in vivo. (A) Sections from the proximal aorta were stained for immunohistochemistry with an antibody against activated phosphorylated Ser ${ }^{473}$-Akt or an IgG control antibody. Medial and intimal areas are indicated. Areas within dotted outlines were enlarged to show the phosphorylated Akt-immunopositive nuclei in the intimal area (arrows). (B) Immunopositive nuclei were quantified in the intimal areas of the lesions from duplicate stained sections and averaged. Data are expressed as the total number of immunopositive nuclei per total lesion area $\left(n=4\right.$ per genotype). ${ }^{*} P<0.05$ versus $p 38 a^{t / / t} A p o e^{-/}$, Mann-Whitney U test). (C) Mouse lesions were also stained for phosphorylated Akt by immunofluorescence or for macrophages in adjacent sections. Shown are representative images of phosphorylated Akt (green) or macrophage staining (red) overlaid with DAPI-stained nuclei. Scale bars: $50 \mu \mathrm{m}$.

Blood Center). The buffy coat was then diluted at a 1:1 ratio with $1 \mathrm{mM}$ EDTA in PBS. The leukocytes were then overlaid gently on a Histopaque cushion (product no. H8889; Sigma-Aldrich) and centrifuged for $20 \mathrm{~min}$ at $400 \mathrm{~g}$ at room temperature. The monocytes were collected at the interface and diluted 10 -fold in plain RPMI medium. The cells were centrifuged for $10 \mathrm{~min}$ at $200 \mathrm{~g}$ and washed this way an additional 2 times. After the final spin, the pellet was resuspended in $20 \mathrm{ml}$ complete medium (20\% human serum, $1 \% \mathrm{~L}$-glutamine, $1 \%$ penicillin/streptomycin, and $1 \mathrm{ng} / \mathrm{ml} \mathrm{GM-CSF}$ ). The cells were then plated at $1.5 \mathrm{ml} /$ well in 24-well cell culture plates, and the medium was changed every $4 \mathrm{~d}$ until day 10 .

In situ TUNEL and macrophage apoptosis assays. Apoptotic cells in the inti$\mathrm{mal}$ area of atherosclerotic lesions were labeled after proteinase $\mathrm{K}$ treatment by TUNEL using the in situ cell death detection kit TMR-red (Roche
Diagnostics) according to the manufacturer's protocol. Only TUNEL-positive cells that colocalized with DAPI-stained nuclei were counted as being positive. For in vitro analysis, macrophages were assayed for early to midstage apoptosis by staining with Alexa Fluor 488-conjugated Annexin V (green) and for late-stage apoptosis by costaining with propidium iodide (red), as described previously (2). TUNEL and annexin staining were viewed at room temperature using an Olympus IX-70 inverted fluorescent microscope equipped with a mercury 100-W lamp (CHIU Technical Corp.), filter wheels, fluorescent filters (Chroma), an Olympus LCPlanF1 $\times 20$ objective, DP Manager Basic imaging software (version 3.1; Olympus), and an Olympus DP71 CCD camera. Representative fields (4-6 fields containing approximately 1,000 cells) were photographed for each condition for the annexin assay. The number of Annexin V-or PI-positive cells were counted 


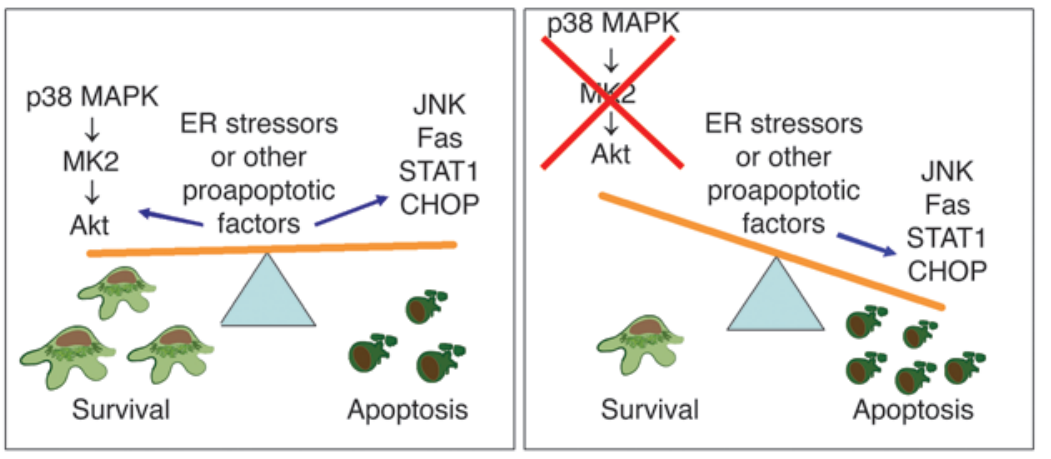

Figure 9

Role of p38 MAPK in protecting macrophages from apoptosis in atherosclerotic lesions. Macrophage death can be triggered by various proapoptotic events, including ER stress factors, cytokines, and Fas, that work alone or, most likely, in combination to trigger macrophage death. In vivo evidence has supported an important role for STAT1 and the ER stress pathways in promoting macrophage apoptosis and advanced plaque necrosis $(15,16,19)$. Furthermore, Fas, JNK, STAT1, and CHOP are all necessary for ER stress-mediated apoptosis in vitro $(2,15,18,27,63)$. Activation of the p38-MK2-Akt pathway appears to play an essential role in balancing the survival-death pathway by protecting from macrophage apoptosis and necrotic core formation. When the p38-Akt survival pathway is lost, macrophage apoptosis is increased. See Discussion for details.

and expressed as a percent of the total number of cells in at least 4 separate fields from duplicate wells. For TUNEL analysis, DAPI and TUNEL images were merged using Photoshop CS analysis software (version 8.0; Adobe), and the number of TUNEL- and DAPI-positive cells was counted from each area of the lesion.

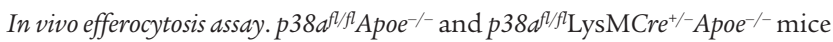
were given an i.p. injection of concanavalin A to elicit the macrophages. The experiment was started $3 \mathrm{~d}$ later. Jurkat $\mathrm{T}$ cells were labeled for $2 \mathrm{~h}$ with $1 \mu \mathrm{g} / \mathrm{ml}$ Calcein-AM (Invitrogen). The cells were centrifuged at $300 \mathrm{~g}$ for $3 \mathrm{~min}$ and washed in RPMI. UV-induced apoptotic Jurkat cells were generated by irradiating the cells for $10 \mathrm{~min}\left(254 \mathrm{~nm}, 20 \mathrm{~J} / \mathrm{cm}^{2}\right)$. After $3 \mathrm{~h}$, the apoptotic cells were collected and resuspended in PBS. We injected $15 \times 10^{6}$ apoptotic cells into each mouse. After $30 \mathrm{~min}$, the mouse was sacrificed, and the peritoneal leukocytes were harvested by lavage. Macrophages were centrifuged and resuspended in PBS and were labeled with PE-conjugated anti-mouse macrophage F4/80 (Cedarlane) for $30 \mathrm{~min}$ in the dark on ice. The cells were centrifuged, washed once, and fixed in $4 \%$ paraformaldehyde for $10 \mathrm{~min}$. Again, cells were centrifuged and resuspended in $1 \mu \mathrm{g} / \mathrm{ml}$ DAPI in PBS to stain the nuclei. Next, cells were centrifuged, washed once, and resuspended in $500 \mu \mathrm{l}$ PBS and stored in the dark at $4^{\circ} \mathrm{C}$. Cells were analyzed and imaged by fluorescence microscopy.

Adenovirus transduction. Adenovirus vectors encoding Lac $\mathrm{Z}$ and Myr-Akt were previously described (62). Amplified adenovirus vectors were purified with Vivapure AdenoPACK 100 (Sartorius) and titrated with Adeno-X Rapid Titer Kit (BD Biosciences). Concanavalin A-elicited or bone marrow-derived macrophages were infected at $90 \%$ confluency with adenovirus for $18 \mathrm{~h}$. MOI of 500 was used in all experiments, and transduction was performed in full medium (DMEM supplemented with 10\% FBS and 20\% L cell-conditioned medium plus $1 \%$ penicillin/streptomycin/glutamine).

Immunoblot analysis. Cells were lysed in a buffer containing $2 \%$ SDS, $62.5 \mathrm{mM}$ Tris-HCl (pH 6.8), 10\% glycerol, $50 \mathrm{mM} \mathrm{DTT}$, and $0.01 \%$ bromphenol blue and boiled at $100^{\circ} \mathrm{C}$ for $5 \mathrm{~min}$. Approximately $100 \mu \mathrm{g}$ lysate protein was separated on a 4\%-20\% gradient SDS-PAGE gel (Invitrogen) and electrotransferred to $0.45-\mu \mathrm{m}$ nitrocellulose membrane using a BioRad mini-transfer tank. Membranes were incubated with primary antibodies overnight, and the protein bands were detected with horseradish peroxidase-conjugated secondary antibodies and Supersignal West Pico enhanced chemiluminescent solution (Pierce). Membranes were stripped with Restore Western Blot Stripping Buffer (Pierce) for $15 \mathrm{~min}$ at room temperature and immunoblotted with antibodies to actin or total Akt to control for differences in loading.

Statistics. Values are mean \pm SEM unless otherwise indicated. $n$ is noted in the figure legends. Absent error bars in graphs signify SEM values smaller than the graphic symbols. Comparison of mean values between groups was evaluated by 2 -tailed Student's $t$ test, Mann-Whitney $U$ test, or ANOVA. A $P$ value less than 0.05 was considered significant.

\section{Acknowledgments}

This work was supported by NIH grant HL079801-02 and American Heart Association Scientist Development Grant 0735594T to T. Seimon, and by NIH grants HL087123 and HL075662 and US Army Medical Research and Materiel Command grant W81XWH06-1-0212 to I.A. Tabas. The authors thank Anton Seimon for help with the data entry and preparation and Connie Woo for assistance with some of the cell culture experiments.

Received for publication August 25, 2008, and accepted in revised form February 4, 2009.

Address correspondence to: Tracie A. Seimon, Department of Medicine, Division of Molecular Medicine, Columbia University, PH 9-405, 630 W. 168th Street, New York, New York 10032, USA. Phone: (212) 305-5669; Fax: (212) 305-5052; E-mail: tad2105@columbia.edu.
1. Schindler, J.F., Monahan, J.B., and Smith, W.G. 2007. p38 pathway kinases as anti-inflammatory drug targets. J. Dent. Res. 86:800-811.

2. DeVries-Seimon, T., et al. 2005. Cholesterolinduced macrophage apoptosis requires ER stress pathways and engagement of the type A scavenger receptor. J. Cell Biol. 171:61-73.

3. Jagavelu, K., et al. 2007. Systemic deficiency of the MAP kinase activated protein kinase 2 reduces atherosclerosis in hypercholesterolemic mice. Circ. Res. 101:1104-1112.

4. Proctor, B.M., et al. 2008. Requirement for p38 mitogen-activated protein kinase activity in neointima formation after vascular injury. Circulation. 118:658-666.

5. Matsuzawa, A., and Ichijo, H. 2001. Molecular mechansims of the decision between life and death: regulation of apoptosis signal regulating kinase 1. 
J. Biochem. 130:1-8.

6. Lee, J.C., et al. 1994. A protein kinase involved in the regulation of inflammatory cytokine biosynthesis. Nature. 372:739-746.

7. Badger, A.M., et al. 1996. Pharmacological profile of SB 203580 , a selective inhibitor of cytokine suppressive binding protein/p38 kinase, in animal models of arthritis, bone resorption, endotoxin shock and immune function. J. Pharmacol. Exp. Ther. 279:1453-1461.

8. Kang, Y.J., et al. 2008. Macrophage deletion of p38\{alpha\} partially impairs lipopolysaccharide-induced cellular activation. J. Immunol. 180:5075-5082.

9. Kim, C., et al. 2008. The kinase p38[alpha] serves cell type-specific inflammatory functions in skin injury and coordinates pro- and anti-inflammatory gene expression. Nat. Immunol. 9:1019-1027.

10. Kolodgie, F.D., et al. 2004. Pathologic assessment of the vulnerable human coronary plaque. Heart. 90:1385-1391.

11. Libby, P., et al. 1996. Macrophages and atherosclerotic plaque stability. Curr. Opin. Lipidol. 7:330-335.

12. Tabas, I. 2005. Consequences and therapeutic implications of macrophage apoptosis in atherosclerosis: the importance of lesion stage and phagocytic efficiency. Arterioscler. Thromb. Vasc. Biol. 25:2255-2264.

13. Gough, P.J., Gomez, I.G., Wille, P.T., and Raines, E.W. 2006. Macrophage expression of active MMP9 induces acute plaque disruption in apoE-deficient mice. J. Clin. Invest. 116:59-69.

14. Gargalovic, P.S., et al. 2006. The unfolded protein response is an important regulator of inflammatory genes in endothelial cells. Arterioscler. Thromb. Vasc. Biol. 26:2490-2496.

15. Lim, W.S., et al. 2008. STAT1 is critical for apoptosis in macrophages subjected to endoplasmic reticulum stress in vitro and in advanced atherosclerotic lesions in vivo. Circulation. 117:940-951.

16. Myoishi, M., et al. 2007. Increased endoplasmic reticulum stress in atherosclerotic plaques associated with acute coronary syndrome. Circulation. 116:1226-1233.

17. Zhou, J., Lhotak, S., Hilditch, B.A., and Austin, R.C. 2005. Activation of the unfolded protein response occurs at all stages of atherosclerotic lesion development in apolipoprotein E-deficient mice. Circulation. 111:1814-1821.

18. Feng, B., et al. 2003. The endoplasmic reticulum is the site of cholesterol-induced cytotoxicity in macrophages. Nat. Cell Biol. 5:781-792.

19. Feng, B., et al. 2003. Niemann-Pick C heterozygosity confers resistance to lesional necrosis and macrophage apoptosis in murine atherosclerosis. Proc. Natl. Acad. Sci. U. S. A. 100:10423-10428.

20. Seimon, T., and Tabas, I. 2008. Mechanisms and consequences of macrophage apoptosis in atherosclerosis. J. Lipid Res. Online publication ahead of print. doi:10.1194/jlr.R800032-JLR200.

21. Schrijvers, D.M., De Meyer, G.R., Herman, A.G., and Martinet, W. 2007. Phagocytosis in atherosclerosis: Molecular mechanisms and implications for plaque progression and stability. Cardiovasc. Res. 73:470-480.

22. Bucciarelli, L.G., et al. 2002. RAGE blockade stabilizes established atherosclerosis in diabetic apolipoprotein E-null mice. Circulation. 106:2827-2835

23. Li, J., and Holbrook, N.J. 2004. Elevated gadd153/ chop expression and enhanced c-Jun $\mathrm{N}$-terminal protein kinase activation sensitizes aged cells to ER stress. Exp. Gerontol. 39:735-744.

24. Park, J.M., Greten, F.R., Li, Z.W., and Karin, M. 2002. Macrophage apoptosis by anthrax lethal factor through p38 MAP kinase inhibition. Science. 297:2048-2051.
25. Wang, Y., et al. 2007. The role of the NADPH oxidase complex, p38 MAPK, and Akt in regulating human monocyte/macrophage survival. Am. J. Respir. Cell Mol. Biol. 36:68-77.

26. Li, Y., et al. 2004. Enrichment of endoplasmic reticulum with cholesterol inhibits SERCA2b activity in parallel with increased order of membrane lipids. Implications for depletion of ER calcium stores and apoptosis in cholesterol-loaded macrophages. J. Biol. Chem. 279:37030-37039.

27. Seimon, T.A., et al. 2006. Combinatorial pattern recognition receptor signaling alters the balance of life and death in macrophages. Proc. Natl. Acad. Sci. U. S. A. 103:19794-19799.

28. Adams, R.H., et al. 2000. Essential role of p38[alpha] MAP kinase in placental but not embryonic cardiovascular development. Mol. Cell. 6:109-116.

29. El-Shafei, A., and Kern, M. 2002. New techniques for the evaluation of the vulnerable plaque. J. Invasive Cardiol. 14:129-137.

30. Kockx, M.M. 1998. Apoptosis in the atherosclerotic plaque: quantitative and qualitative aspects. Arterioscler. Thromb. Vasc. Biol. 18:1519-1522.

31. Kockx, M.M., and Herman, A.G. 1998. Apoptosis in atherogenesis: implications for plaque destabilization. Eur. Heart J. 19(Suppl. G):G23-G28.

32. Terasaka, N., Wang, N., Yvan-Charvet, L., and Tall, A.R. 2007. High-density lipoprotein protects macrophages from oxidized low-density lipoproteininduced apoptosis by promoting efflux of 7-ketocholesterol via ABCG1. Proc. Natl. Acad. Sci. U. S. A. 104:15093-15098.

33. Voccoli, V., Mazzoni, F., Garcia-Gil, M., and Colombaioni, L. 2007. Serum-withdrawal-dependent apoptosis of hippocampal neuroblasts involves $\mathrm{Ca}++$ release by endoplasmic reticulum and caspase-12 activation. Brain Res. 1147:1-11.

34. Jinlian, L., Yingbin, Z., and Chunbo, W. 2007. p38 MAPK in regulating cellular responses to ultraviolet radiation. J. Biomed. Sci. 14:303-312.

35. Hsu, L.C., et al. 2004. The protein kinase PKR is required for macrophage apoptosis after activation of Toll-like receptor 4. Nature. 428:341-345.

36. Park, J.M., et al. 2005. Signaling pathways and genes that inhibit pathogen-induced macrophage apoptosis--CREB and NF-kappaB as key regulators. Immunity. 23:319-329.

37. Rane, M.J., et al. 2001. p38 Kinase-dependent MAPKAPK-2 activation functions as 3-phosphoinositidedependent kinase-2 for AKT in human neutrophils. J. Biol. Chem. 276:3517-3523.

38. Rane, M.J., et al. 2003. Heat shock protein 27 controls apoptosis by regulating Akt activation. J. Biol. Chem. 278:27828-27835.

39. Taniyama, Y., et al. 2004. Role of p38 MAPK and MAPKAPK-2 in angiotensin II-induced Akt activation in vascular smooth muscle cells. Am. J. Physiol. Cell Physiol. 287:C494-C499.

40. Wu, R., et al. 2007. Hsp27 regulates Akt activation and polymorphonuclear leukocyte apoptosis by scaffolding MK2 to Akt signal complex. J. Biol. Chem. 282:21598-21608.

41. Hosoi, T., Hyoda, K., Okuma, Y., Nomura, Y., and Ozawa, K. 2007. Akt up- and down-regulation in response to endoplasmic reticulum stress. Brain Res. 1152:27-31.

42. Hu, P., Han, Z., Couvillon, A.D., and Exton, J.H. 2004. Critical role of endogenous Akt/IAPs and MEK1/ERK pathways in counteracting endoplasmic reticulum stress-induced cell death. J. Biol. Chem. 279:49420-49429.

43. Trotman, L.C., et al. 2006. Identification of a tumour suppressor network opposing nuclear Akt function. Nature. 441:523-527.

44. Tsujita, Y., et al. 2006. Nuclear targeting of Akt antagonizes aspects of cardiomyocyte hypertrophy. Proc. Natl. Acad. Sci. U. S. A. 103:11946-11951.

45. Dickhout, J.G., et al. 2005. Peroxynitrite causes endoplasmic reticulum stress and apoptosis in human vascular endothelium: implications in atherogenesis. Arterioscler. Thromb. Vasc. Biol. 25:2623-2629.

46. Han, S., et al. 2006. Macrophage insulin receptor deficiency increases ER stress-induced apoptosis and necrotic core formation in advanced atherosclerotic lesions. Cell Metab. 3:257-266.

47. Werstuck, G.H., et al. 2006. Glucosamine-induced endoplasmic reticulum dysfunction is associated with accelerated atherosclerosis in a hyperglycemic mouse model. Diabetes. 55:93-101.

48. Borradaile, N.M., et al. 2006. Disruption of endoplasmic reticulum structure and integrity in lipotoxic cell death. J. Lipid Res. 47:2726-2737.

49. Thuerauf, D.J., et al. 2006. Activation of the unfolded protein response in infarcted mouse heart and hypoxic cultured cardiac myocytes. Circ. Res. 99:275-282.

50. Werstuck, G.H., et al. 2001. Homocysteine-induced endoplasmic reticulum stress causes dysregulation of the cholesterol and triglyceride biosynthetic pathways. J. Clin. Invest. 107:1263-1273.

51. Lupu, F., Danaricu, I., and Simionescu, N. 1987. Development of intracellular lipid deposits in lipid-laden cells of atherosclerotic lesions. A cytochemical and ultrastructural study. Atherosclerosis. 67:127-142.

52. Manning-Tobin, J.J., et al. 2008. Loss of SR-A and CD36 activity reduces atherosclerotic lesion complexity without abrogating foam cell formation in hyperlipidemic mice. Arterioscler. Thromb. Vasc. Biol. 29:19-26.

53. Fernandez-Hernando, C., et al. 2007. Loss of akt 1 leads to severe atherosclerosis and occlusive coronary artery disease. Cell Metab. 6:446-457.

54. Kotlyarov, A., et al. 2002. Distinct cellular functions of MK2. Mol. Cell. Biol. 22:4827-4835.

55. Morris, J.B., et al. 2008. p38 MAPK inhibition reduces aortic ultrasmall superparamagnetic iron oxide uptake in a mouse model of atherosclerosis: MRI assessment. Arterioscler. Thromb. Vasc. Biol. 28:265-271.

56. Ross, A.C., Go, K.J., Heider, J.G., and Rothblat, G.H. 1984. Selective inhibition of acyl coenzyme A: cholesterol acyltransferase by compound 58-035. J. Biol. Chem. 259:815-819.

57. Havel, R.J., Eder, H., and Bragdon, J. 1955. The distribution and chemical composition of ultracentrifugally reported lipoproteins in human serum. J. Clin. Invest. 34:1345-1353.

58. Basu, S.K., Goldstein, J.L., Anderson, R.G.W., and Brown, M.S. 1976. Degradation of cationized low density lipoprotein and regulation of cholesterol metabolism in homozygous familial hypercholesterolemia fibroblasts. Proc. Natl. Acad. Sci. U. S. A. 73:3178-3182.

59. Clausen, B.E., Burkhardt, C., Reith, W., Renkawitz, R., and Forster, I. 1999. Conditional gene targeting in macrophages and granulocytes using LysMcre mice. Transgenic Res. 8:265-277.

60. Engel, F., et al. 2005. p38 MAP kinase inhibition enables proliferation of adult mammalian cardiomyocytes. Genes Devel. 19:1175-1187.

61. Cook, A.D., Braine, E.L., and Hamilton, J.A. 2003. The phenotype of inflammatory macrophages is stimulus dependent: implications for the nature of the inflammatory response. J. Immunol. 171:4816-4823.

62. Nakae, J., Kitamura, T., Ogawa, W., Kasuga, M., and Accili, D. 2001. Insulin regulation of gene expression through the forkhead transcription factor Foxo1 (Fkhr) requires kinases distinct from Akt. Biochemistry. 40:11768-11776.

63. Yao, P.M., and Tabas, I. 2000. Free cholesterol loading of macrophages induces apoptosis involving the fas pathway. J. Biol. Chem. 275:23807-23813. 